\title{
La délégation des tâches au niveau communautaire dans le domaine de la planification familiale dans les pays du Partenariat de Ouagadougou : Expériences et leçons apprises pour une mise en œuvre effective-Rapport de synthèse
}

\author{
Fatou Mbow \\ Population Council \\ El Hadji Alioune Badara Ningue \\ Nafissatou Diop \\ Population Council \\ Babacar Mane \\ Population Council
}

Rodrigue Ngouana

Follow this and additional works at: https://knowledgecommons.popcouncil.org/departments_sbsr-rh How does access to this work benefit you? Let us know!

\section{Recommended Citation}

Mbow, Fatou, El Hadji Alioune Badara Ningue, Nafissatou Diop, Babacar Mane, and Rodrigue Ngouana. 2015. "La délégation des tâches au niveau communautaire dans le domaine de la planification familiale dans les pays du Partenariat de Ouagadougou : Expériences et leçons apprises pour une mise en œuvre effective-Rapport de synthèse." Dakar: Population Council. 
LA DELEGATION DES TACHES EN MATIERE DE PLANIFICATION FAMILIALE AU NIVEAU COMMUNAUTAIRE DANS LES PAYS DU PARTENARIAT DE OUAGADOUGOU : EXPERIENCES ET LEÇONS APPRISES POUR UNE MISE EN OEUVRE EFFECTIVE RAPPORT DE SYNTHESE

Fatou Bintou Mbow El Hadji Alioune Badara Ningue Nafissatou Diop Babacar Mané Rodrigue Ngouana 


\section{ir pouvarion \\ Ideas. Evidence. Impact.}

Le Population Council s'attelle aux questions cruciales de santé et de développement - qu'il s'agisse d'endiguer la progression du VIH, d'améliorer la santé reproductive et d'offrir aux jeunes la promesse d'une vie satisfaisante et productive. Par ses travaux de recherche en biomédecine, en sciences sociales et en santé publique dans 50 pays, il s'efforce d'apporter avec ses partenaires des solutions qui conduisent à des politiques, des programmes et des technologies plus efficaces, pour améliorer la vie partout dans le monde. Fondé en 1952, le Population Council est une organisation non gouvernementale à but non lucratif. II siège à New York sous la conduite d'un conseil d'administration international.

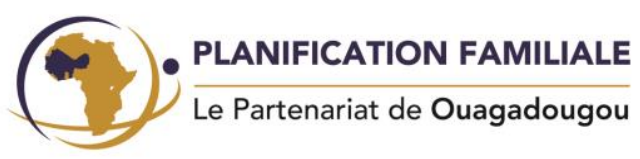

Le Partenariat de Ouagadougou a été lancé en février 2011, lors de la Conférence régionale sur la Population, le Développement et la Planification Familiale : I'urgence d'agir, qui s'était tenue dans la capitale du Burkina Faso. II mise sur l'engagement des gouvernements, une meilleure coordination entre les bailleurs de fonds pour optimiser leurs soutiens aux pays, une accélération de la mise en œuvre des interventions à haut impact et également sur une collaboration et coopération aux plans national et régional pour remédier au taux élevé des besoins non satisfaits en matière de planification familiale. Le Partenariat de Ouagadougou compte neuf pays membres : Bénin, Burkina Faso, Côte d’Ivoire, Guinée, Mali, Mauritanie, Niger, Sénégal et Togo.

Citation recommandée: Mbow FB, Ningue EAB, Diop N, Mané B, Ngouana R. 2015. "La délégation des tâches au niveau communautaire dans le domaine de la planification familiale dans les pays du Partenariat de Ouagadougou : Expériences et leçons apprises pour une mise en œuvre effective - Rapport de synthèse". Dakar: Population Council. 


\section{Table des matières}

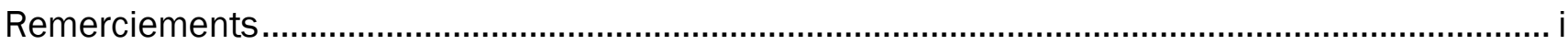

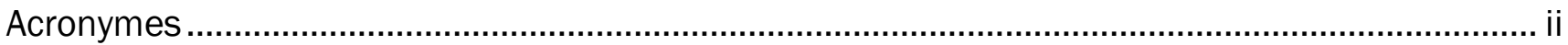

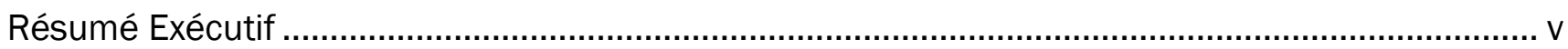

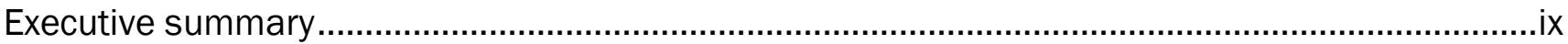

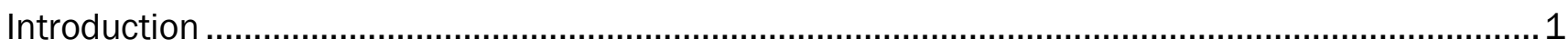

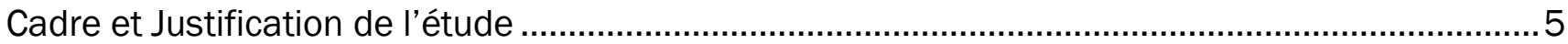

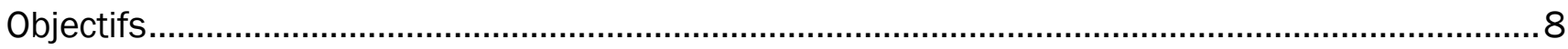

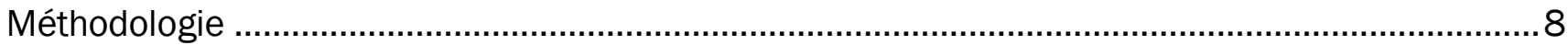

Environnement Politique, légal et règlementaire ..................................................................

Expériences de délégation des tâches par pays ……………...................................................15

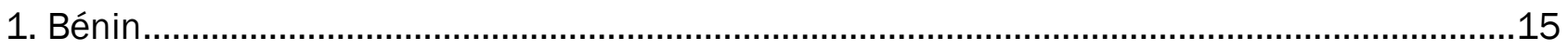

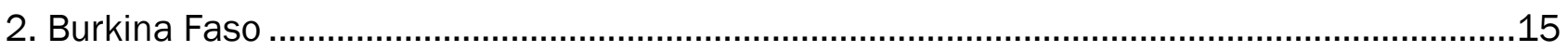

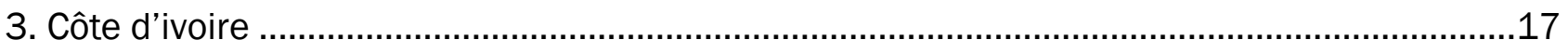

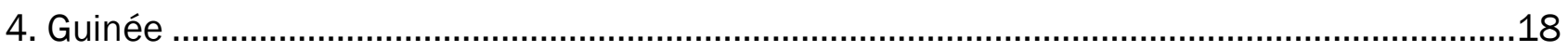

5. Mali

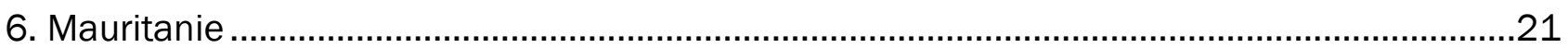

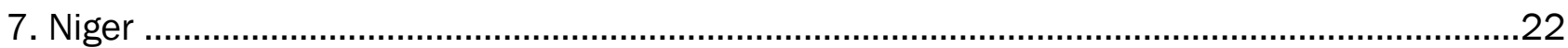

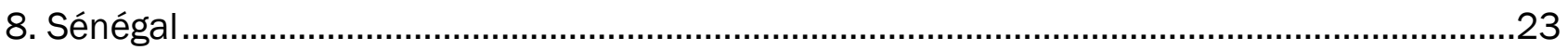

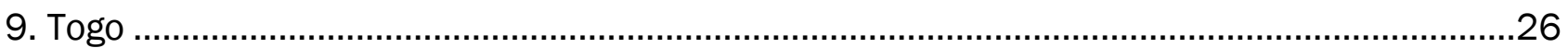

Tableau synoptique des méthodes contraceptives offertes par les agents de santé communautaires dans les pays du Partenariat de Ouagadougou .............................................27

Contraintes à la mise en œuvre des activités de délégation des tâches : ..................................29

Leçons apprises ................................................................................................................

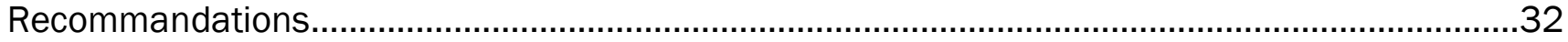

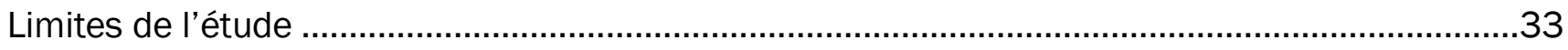

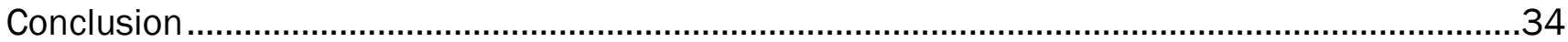

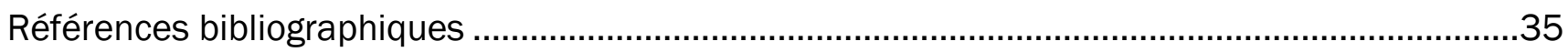





\section{Remerciements}

Les auteurs remercient toutes les organisations, institutions et personnes qui ont eu à contribuer à la réalisation de cette étude en facilitant l'accès à la documentation disponible ou en orientant vers d'autres parties prenantes susceptibles de détenir de la documentation utile. 


\section{Acronymes}

\begin{tabular}{|c|c|}
\hline ABBEF & Association Burkinabé pour le Bien-être Familial \\
\hline ACDEV & Action et Développement \\
\hline ACO & Afrique Centrale et Occidentale \\
\hline ADBC & Agent de Distribution à Base Communautaire \\
\hline ADESCO & Appui au Développement à la Santé Communautaire \\
\hline AGBEF & Association Guinéenne pour le Bien-Etre Familial \\
\hline AMPF & Association Mauritanienne pour la promotion de la famille \\
\hline ANIMAS & Agence Nigérienne de Marketing Social \\
\hline ANSD & Agence Nationale de la Statistique et de la Démographie \\
\hline ASACO & Association de Santé Communautaire \\
\hline ASAPSU & Association de Soutien à I'Autopromotion Sanitaire Urbain \\
\hline ASBC & Agent de Santé à Base Communautaire \\
\hline ASBEF & Association Sénégalaise pour le Bien-Etre Familial \\
\hline ASC & Agent de santé Communautaire \\
\hline ASV & Agent de Santé Villageois \\
\hline ATBEF & Association Togolaise pour le Bien-Etre Familial \\
\hline $\mathrm{CCC}$ & Communication pour le Changement de Comportement \\
\hline CEDEAO & Communauté Economique des Etats de l'Afrique de l'Ouest \\
\hline CEFOREP & Centre Régional de Formation, de Recherche et de plaidoyer en Santé de la Reproduction \\
\hline CIPD & Conférence Internationale sur la Population et le Développement \\
\hline C-PIHI & Paquet d'Intervention à Haut Impact Communautaire \\
\hline CSCOM & Centre de Santé Communautaire \\
\hline CSI & Centre de Santé Intégré \\
\hline CSPS & Centre de Santé et de Promotion Sociale \\
\hline DBC & Distribution à Base Communautaire \\
\hline DHPES & Direction de l'Hygiène Publique et de l'Education pour la Santé \\
\hline DIU & Dispositif Intra Utérin \\
\hline DNS & Direction Nationale de la Santé \\
\hline DPS & Direction de la Promotion de la Santé \\
\hline DRS & Direction Régionale de la Santé \\
\hline DRSP & Direction Régionale de la Santé Publique \\
\hline DSCMP & Direction de la Santé Communautaire et de la Médecine de Proximité \\
\hline
\end{tabular}




\begin{tabular}{|c|c|}
\hline DSFC & Division Santé Familiale et Communautaire \\
\hline DSME & Direction de la Santé de la Mère et de l’Enfant \\
\hline DSRSE & Direction de la Santé de la Reproduction et de la Survie de l'Enfant \\
\hline ECD & Equipe Cadre de District \\
\hline EDS & Enquête Démographique et de Santé \\
\hline EDS MICS & Enquête Démographique et de Santé à Indicateurs Multiples \\
\hline $\mathrm{FCl}$ & Family Care International \\
\hline $\mathrm{FHI}$ & Family Health International \\
\hline $\mathrm{GIZ}$ & Coopération Internationale Allemande \\
\hline GTZ & Coopération Technique Allemande \\
\hline $\mathrm{HKI}$ & Hellen Keller International \\
\hline ICM & International Confederation of Midwives \\
\hline $\mathrm{ICP}$ & Infirmier Chef de Poste \\
\hline IEC & Information Education et Communication \\
\hline IM & Intra Musculaire \\
\hline IPPF & Fédération Internationale pour la Planification Familiale \\
\hline ISF & Indice Synthétique de Fécondité \\
\hline JHU & Johns Hopkins University \\
\hline MAMA & Méthode de l'Allaitement Maternel et de l'Aménorrhée \\
\hline MCHIP & Programme Intégré de Santé Maternelle et Infantile \\
\hline MDM & Médecin Du Monde \\
\hline MSAS & Ministère de la Santé et de l'Action Sociale \\
\hline MSH & Management Sciences for Health \\
\hline MSHP & Ministère de la Santé et de l'Hygiène Publique \\
\hline MSP & Ministère de la Santé Publique \\
\hline OBCE & Organisation à Base Communautaire d'Exécution \\
\hline OIP & Offre Initiale de Pilule \\
\hline OMD & Objectif du Millénaire pour le Développement \\
\hline OMS & Organisation Mondiale de la Santé \\
\hline OOAS & Organisation Ouest Africaine pour la Santé \\
\hline PAA & Plan Annuel des Activités \\
\hline PADS & Programme d'Appui au Développement du Secteur de la Santé \\
\hline
\end{tabular}




\begin{tabular}{|c|c|}
\hline PCIMNE-C & Prise en Charge Intégrée des Maladies du Nouveau-né et de l'Enfant au niveau Communautaire \\
\hline PE & Pair Educateur \\
\hline $\mathrm{PF}$ & Planification Familiale \\
\hline PFE & Pratique Familiale Essentielle \\
\hline PNDS & Programme National de Développement Sanitaire \\
\hline PNP & Politiques Normes et Protocoles \\
\hline PRISM & Pour Renforcer les Interventions en Santé de la reproduction et MST/SIDA \\
\hline PROSAD & Programme Santé Sexuelle, Droits Humains \\
\hline PSI & Population Services International \\
\hline PSSC & Programme Santé de I'USAID/Santé Communautaire \\
\hline PTA & Plan de Travail Annuel \\
\hline PTF & Partenaire Technique et Financier \\
\hline PUSKC & Programme USAID Santé Kènèya Ciwara \\
\hline RAP & Recherche Action Plaidoyer \\
\hline $\mathrm{RC}$ & Relais Communautaires \\
\hline SBC & Services de santé à Base Communautaire \\
\hline SECONAF & Sécurité Contraceptive en Afrique Francophone \\
\hline SMN & Santé Maternelle et Néonatale \\
\hline SR & Santé de la Reproduction \\
\hline SSR & Santé Sexuelle et Reproductive \\
\hline STEP UP & Strengthening Evidence for Programming on Unintended Pregnancy \\
\hline TIDC & Traitement par l'Ivermectine sous directive communautaire \\
\hline UCPO & Unité de Coordination du Partenariat de Ouagadougou \\
\hline UNFPA & Fonds de Nations Unies pour la Population \\
\hline UNICEF & Fonds de Nations Unies pour l'Enfance \\
\hline USAID & Agence Américaine pour le Développement International \\
\hline USP & Unité de Soins Périphérique \\
\hline VAD & Visite à Domicile \\
\hline VIH/SIDA & Virus Immunodéficience Humaine/Syndrome Immunodéficience Acquis \\
\hline
\end{tabular}




\section{Résumé Exécutif}

En Afrique de l'Ouest, les pays francophones présentent les plus forts taux de mortalité maternelle et infantojuvénile, les indices synthétiques de fécondité les plus élevés et les taux de prévalence contraceptive les plus faibles.

De nombreuses conférences ont permis aux pays participants, notamment ceux d'Afrique, de s'accorder sur des stratégies communes en matière de santé de la reproduction (SR), y compris la planification familiale (PF), et de leur octroyer un rang de priorité dans l'élaboration des politiques et programmes. Afin de faciliter la disponibilité et l'accessibilité de la PF, plusieurs initiatives ont été développées, parmi lesquelles la Conférence Régionale sur le thème: "Population, Développement et Planification Familiale en Afrique de l'Ouest Francophone : I'urgence d'agir " tenue à Ouagadougou au Burkina Faso en 2011. Cette conférence a été une étape déterminante pour opérationnaliser cette approche concertée des questions de SR. Elle a permis la création du "Partenariat de Ouagadougou " (PO) qui regroupe neuf (9) pays francophones d'Afrique de l'Ouest (Bénin, Burkina Faso, Côte d'Ivoire, Guinée, Mali, Mauritanie, Niger, Sénégal et Togo) et leurs partenaires techniques et financiers. Ce réseau vise à accélérer les progrès dans I'utilisation des services de PF dans ces pays. Son objectif principal est de recruter au moins un million de nouvelles utilisatrices de méthodes contraceptives en 2015.

\section{Cadre et justification}

Selon l'Organisation Mondiale de la Santé (OMS), il y a un déficit global du personnel de santé qualifié de plus de quatre (4) millions de personnes (OMS, 2008). Cette situation est particulièrement critique dans les pays en voie de développement. Selon la publication "Accroître l'accès aux personnels de santé dans les zones rurales ou reculées grâce à une meilleure fidélisation : recommandations pour une politique mondiale " (OMS, 2010), la moitié environ de la population mondiale vit dans des zones rurales, alors que celles-ci ne sont desservies que par 38 pour cent de l'effectif infirmier total et moins du quart de l'effectif des médecins (24 pour cent). La situation est particulièrement dramatique dans 57 pays où la pénurie de professionnels de santé qualifiés est telle qu'on estime à un milliard le nombre des personnes privées de tous services de soins de santé essentiels.

Pour pallier cette carence en ressources humaines et afin d'assurer un meilleur accès aux services de santé de base aux communautés, une des stratégies identifiées et expérimentée est le recours aux Agents de Santé Communautaires (ASC), à travers le transfert/partage de tâches (délégation des tâches). Ainsi, pour aider les pays à optimiser les performances des agents de santé, l'OMS a rédigé une série complète de recommandations pour le partage des tâches, notamment pour la fourniture de contraceptifs (OMS, 2012).

Cette étude permet d'évaluer les expériences passées et les efforts en cours dans les pays du PO pour adresser le déficit de main d'œuvre en vue d'améliorer l'accès aux services de santé au niveau communautaire. Elle a pour objectif de documenter les expériences et leçons apprises sur cette approche de délégation des tâches dans les pays du Partenariat.

\section{Méthodologie}

Cette recherche a consisté à examiner et à analyser toute la documentation disponible sur les expériences passées et efforts en cours en matière de délégation des tâches dans le domaine de la PF, particulièrement celles menées au niveau communautaire avec du personnel non médical. Une fiche de collecte standard a été développée pour systématiser et harmoniser la collecte des informations disponibles pour chaque pays. En 
plus de la revue de la littérature sur Internet, tous les points focaux du PO et toutes les parties prenantes connues ont été contactées pour collecter la documentation disponible à leur niveau ou pour apporter des compléments d'information. L'analyse a combiné une démarche descriptive, qui a pour objet de décrire, et une démarche inductive, qui consiste à partir des informations disponibles pour élaborer une analyse théorique. Cela a permis de présenter un état compréhensif des lieux sur la base des axes thématiques dégagés, dans le but d'apprécier les stratégies définies, leur significativité, et les contraintes dans la mise en œuvre.

\section{Environnement politique, légal et règlementaire}

Dans les neuf (9) pays du partenariat, les environnements politiques sont favorables à la PF avec les différents plans de repositionnement de la PF qui ont été élaborés et affinés. L'environnement juridique est également propice à la promotion de la PF avec l'existence d'une loi relative à la santé de la reproduction (loi SR) dans la plupart des pays. En ce qui concerne la délégation des tâches, elle est souvent autorisée à travers des règlements, arrêtés, circulaires ou directives. Cependant, dans la pratique, les tâches dont s'acquittent les agents de santé et autres acteurs au niveau communautaire dépassent souvent ce que la loi leur permet de faire, bien que des restrictions aient aussi été notées.

\section{Expériences de délégation des tâches par pays}

Les expériences de délégation des tâches suivantes ont pu être répertoriées pour chaque pays, bien que de la documentation suffisante n'a pas toujours pu être retrouvée. La plupart des expériences se sont déroulées avec du personnel non médical. Cependant, trois (3) cas avec du personnel qualifié ont pu être retracées au Burkina Faso, au Niger et au Sénégal. Ils sont signalés ci-dessous :

- Bénin: Programme Survie de l'Enfant dans l'Ouémé (PSEO).

- Burkina Faso: Projet Planification Familiale; Projet PRAJA/SR; Projet de Distribution à Base Communautaire des produits contraceptifs non prescriptibles par des pairs éducateurs; Programme Santé Sexuelle, Droits Humains (PROSAD); Projet Promotion des comportements à moindre risque en santé de la reproduction; Projet de plaidoyer dans le cadre de l'initiative Advance Family Planning. Ce dernier projet est celui qui a été mené avec du personnel qualifié (accoucheuses brevetés et infirmiers (ères)).

- Côte d'ivoire: Projet "Les services intégrés de santé sexuelle et de la reproduction de qualité sont disponibles pour répondre aux besoins des populations "; Formation de relais communautaires sur les techniques de CCC par le Programme National SR/PF ; Activités de la Direction de la santé communautaire et de la médecine de proximité (DSCMP).

- Guinée: Projet pilote de distribution communautaire des préservatifs; Projet de santé reproductive et sexuelle communautaire durable en région forestière de la Guinée; Projet PRISM II (Pour Renforcer les Interventions en Santé de la reproduction et MST/SIDA); Initiative Onchocercose-Santé de la Reproduction/Guinée (2006-2012); Développement des services de PF à base communautaire dans 43 Sous-Préfectures et 4 Communes urbaines SONU/MURIGA (Mutuelle des Risques liés à la Grossesse et à l'Accouchement) de 2009 à 2011, en Basse et en Moyenne Guinée ; Projet pilote d'introduction du DepoProvera dans le paquet d'activités des agents de santé communautaire.

- Mali : Projet de DBC de contraceptifs ; Programme USAID Santé Kènèya Ciwara Phases I et II (PUSKC I et PUSKC II); Programme d'Assistance Technique Nationale Plus (ATN Plus); Etude de faisabilité de l'insertion des implants par les matrones.

- Mauritanie : Projet "Etendre l'accès à des services de santé sexuelle et reproductive de qualité ".

- Niger : Projet Planning Familial/Prévention du SIDA; Projet Recherche Action Plaidoyer (RAP) Niger de Médecins Du Monde (MDM). Projet Sayana Press dans la région de Maradi. Ce projet est mené avec du personnel qualifié (agents de santé qualifiés). 
- Sénégal : Counseling initial en PF par des matrones au niveau des points de prestation de services; Projet DBC des contraceptifs de barrière de I'ONG ACDEV; Etude expérimentale sur l'offre de la distribution à base communautaire des services de santé de la reproduction au Sénégal: Une étude de cas dans le district sanitaire de Kébémer; Projet Services à Base Communautaires de Santé de la Reproduction (SBC/SR); Programme de Santé de I'USAID/Santé Communautaire (PSSC); Offre communautaire de pilules et d'injectables par les relais communautaires des ONG ACDEV et ASBEF. Offre de méthodes à longue durée d'action (MLDA) au niveau des postes de santé. Ce projet a été mené avec du personnel qualifié (infirmiers chefs de poste de santé)

- Togo : Projet PF/PCIMNE-C ; Programme de coopération entre I'UNFPA, I'UNICEF, I'OMS et le Ministère de la Santé et I'ONG ADESCO.

Les contraintes dans la mise en œuvre des activités sont généralement liées à l'insuffisance de motivation, à la faiblesse de la supervision, et à la non-utilisation ou mauvaise utilisation d'outils de gestion.

\section{Leçons apprises}

Pour l'essentiel, l'analyse des différentes expériences de délégation des tâches a permis de relever que dans les pays du Partenariat de Ouagadougou, la distribution à base communautaire (DBC) est un mécanisme essentiel de décentralisation des services SR/PF au niveau communautaire ; les ASC représentent un maillon essentiel pour améliorer l'accès aux MLDA ; quand ils sont bien formés et supervisés, les ASC peuvent offrir adéquatement des services de PF au niveau communautaire ; La fourniture de contraceptifs injectables par les ASC pourrait conduire à l'augmentation de l'utilisation des contraceptifs dans les zones de faible taux de prévalence contraceptive, très souvent caractérisées par des niveaux élevés de besoins non satisfaits (BNS), et un manque d'accès à une large gamme de méthodes et de services cliniques; une bonne motivation maintient les ASC dans leur rôle et améliore la qualité de leurs services.

\section{Recommandations}

Les recommandations formulées ont trait au renforcement de l'élaboration de politiques nationales en faveur de l'introduction, de la poursuite et de l'extension de l'offre de méthodes contraceptives au niveau communautaire, en particulier celle des injectables ; au renforcement de la formation, de la supervision et de la motivation des ASC. Par ailleurs, un approvisionnement continu en médicaments et en produits de PF ainsi qu'une meilleure documentation des expériences et interventions ont été suggérés.

\section{Limites de l'étude}

La principale difficulté rencontrée au cours de cette étude a été l'accès à la documentation. Bien que plusieurs pays aient mentionné des activités de délégation des tâches, peu ou pas du tout de documents ont pu être trouvés. De plus, l'absence de réactivité des acteurs pour obtenir la documentation disponible a parfois aussi été un frein.

\section{Conclusion}

La délégation des tâches se pratique déjà à des degrés divers dans les pays du Partenariat de Ouagadougou. Mais la documentation trouvée concerne surtout les expériences menées au niveau communautaire, et notamment avec du personnel non médical. Ainsi, il est apparu que, dans le contexte Ouest-africain, il est possible pour des bénévoles communautaires bien formés et soutenus, d'assurer une administration efficace 
et à moindre risque des contraceptifs hormonaux. La poursuite et la pérennisation de ces activités dépendent toutefois de l'engagement des gouvernements, des ONG partenaires, et de la communauté internationale à assurer un approvisionnement continu en médicaments et en produits de PF et à soutenir techniquement et financièrement les initiatives. Peu d'expériences avec du personnel qualifié ou dans des structures de santé ont pu être retracées. Est-ce par manque d'expériences de telle délégation de tâches ou par manque de documentation des expériences? La question reste ouverte. 


\section{Executive summary}

In West Africa, French-speaking countries have the highest rates of maternal and child mortality, the highest total fertility rates higher and the lowest contraceptive prevalence rates.

Many conferences have enabled participating countries, particularly those in Africa, to agree on common reproductive health $(\mathrm{RH})$ strategies, including family planning (FP), and to give them a priority ranking in the development of policies and programs. To facilitate FP availability and accessibility, several initiatives have been developed, including the Regional Conference on the theme "Population, Development and Family Planning in Francophone West Africa: the urgency to act" held in Ouagadougou, Burkina Faso in 2011. This conference was a key milestone for the operationalization of this collaborative approach of $\mathrm{RH}$ issues. It enabled the creation of the "Ouagadougou Partnership" (PO) that regroups 9 countries of Francophone West Africa (Benin, Burkina Faso, Ivory Coast, Guinea, Mali, Mauritania, Niger, Senegal and Togo) and their technical and financial partners. This network aims to speed up progress in the use of FP services in these countries. Its main objective is to recruit at least one million new users of contraceptive methods in 2015

\section{Framework and justification:}

According to the World Health Organization (WHO), there is a global deficit of qualified health personnel by more than 4 million people (WHO, 2008). This situation is particularly acute in developing countries. According to the publication "Increasing access to health workers in remote and rural areas through improved retention: global policy recommendations" (WHO, 2010), about half of the world population lives in rural areas, while they are served by 38 percent of the total nurses' workplace and less than a quarter of physicians' workforce (24 percent). The situation is particularly dire in 57 countries where the shortage of skilled health professionals is such that it is estimated than one billion people are deprived of all essential health care services

To overcome this lack of human resources and ensure better access to basic community health services, one of the identified and proven strategies is the use of Community Health Workers (CHW), through task shifting/sharing. Therefore, to help countries improve health workers' performance, WHO has drafted a comprehensive set of recommendations for task-sharing, including the provision of contraceptives (WHO, 2012).

This study assesses past experiences and current efforts in OP countries to address labor shortage for improving access to health services at community level. It aims to document experiences and lessons learned on this approach to task-shifting in the countries of the Partnership.

\section{Methodology}

This research consisted of the review and analysis of all available documentation on past experiences and current task shifting efforts in the field of FP, in particular those conducted at the community level with lay health workers. A standard data collection form was developed to systematize and harmonize the compilation of information available for each country. In addition to perusing literature from the Internet, all OP focal points and known stakeholders were contacted to collect available documentation at their level or to provide additional information. The analysis combined a descriptive approach, which aims to describe, and an inductive approach, which builds on existing information to develop a theoretical analysis. This allowed presenting a comprehensive inventory on the basis of the themes that emerged, in order to assess the strategies defined, their significance, and implementation constraints. 


\section{Political, legal and regulatory environment}

In the 9 countries of the partnership, policy environments are favorable to FP with the various FP repositioning plans that have been developed and refined. The legal environment is also conducive to the promotion of FP with the existence of a law on reproductive health ( $\mathrm{RH}$ law) in most countries. Regarding task shifting, it is often permitted through regulations, bylaws, circulars or directives. However, in practice, the tasks health workers and others stakeholders perform at community level often go beyond what the law allows them to do, although restrictions have also been noted.

\section{Task-shifting experiences by country}

The following task shifting experiences have been complied for each country, although sufficient documentation was not always available. Most experiences were conducted with lay health workers. However, it was possible to track 3 cases involving health workers in Burkina Faso, Niger and Senegal. They are flagged below:

- Bénin: Programme Survie de l'Enfant dans l'Ouémé (PSEO).

- Burkina Faso: Projet Planification Familiale; Projet PRAJA/SR; Projet de Distribution à Base Communautaire des produits contraceptifs non prescriptibles par des pairs éducateurs; Programme Santé Sexuelle, Droits Humains (PROSAD); Projet Promotion des comportements à moindre risque en santé de la reproduction; Projet de plaidoyer dans le cadre de l'initiative Advance Family Planning. This last project is the one conducted with health workers (skilled birth attendant and nurses)

- Côte d'ivoire: Projet "Les services intégrés de santé sexuelle et de la reproduction de qualité sont disponibles pour répondre aux besoins des populations "; Formation de relais communautaires sur les techniques de CCC par le Programme National SR/PF ; Activités de la Direction de la santé communautaire et de la médecine de proximité (DSCMP).

- Guinée: Projet pilote de distribution communautaire des préservatifs; Projet de santé reproductive et sexuelle communautaire durable en région forestière de la Guinée; Projet PRISM II (Pour Renforcer les Interventions en Santé de la reproduction et MST/SIDA); Initiative Onchocercose-Santé de la Reproduction/Guinée (2006-2012); Développement des services de PF à base communautaire dans 43 Sous-Préfectures et 4 Communes urbaines SONU/MURIGA (Mutuelle des Risques liés à la Grossesse et à l'Accouchement) de 2009 à 2011, en Basse et en Moyenne Guinée ; Projet pilote d'introduction du DepoProvera dans le paquet d'activités des agents de santé communautaires.

- Mali : Projet de DBC de contraceptifs ; Programme USAID Santé Kènèya Ciwara Phases I et II (PUSKC I et PUSKC II); Programme d'Assistance Technique Nationale Plus (ATN Plus); Etude de faisabilité de l'insertion des implants par les matrones.

- Mauritanie : Projet Etendre l'accès à des services de santé sexuelle et reproductive de qualité.

- Niger: Projet Planning Familial/Prévention du SIDA; Projet Recherche Action Plaidoyer (RAP) Niger de Médecins Du Monde (MDM); Projet Sayana Press dans la région de Maradi. This projet was conducted with health workers (qualified health agents).

- Sénégal : Counseling initial en PF par des matrones au niveau des points de prestation de services; Projet DBC des contraceptifs de barrière de I'ONG ACDEV; Etude expérimentale sur l'offre de la distribution à base communautaire des services de santé de la reproduction au Sénégal : Une étude de cas dans le district sanitaire de Kébémer; Projet Services à Base Communautaires de Santé de la Reproduction (SBC/SR); Programme de Santé de I'USAID/Santé Communautaire (PSSC); Offre communautaire de pilules et d'injectables par les relais communautaires des ONG ACDEV et ASBEF. Offre de méthodes à longue durée d'action (MLDA) au niveau des postes de santé. This project was conducted with health workers (Head nurses of Health Posts) 
- Togo: Projet PF/PCIMNE-C; Programme de coopération entre I'UNFPA, I'UNICEF, l'OMS et le Ministère de la Santé et l'ONG ADESCO.

Constraints in the implementation of activities are generally related to insufficient incentives, weak supervision, and non-use or misuse of management tools.

\section{Lessons learned}

Essentially, the analysis of the various task shifting experiences has identified that in the Ouagadougou Partnership countries, community-based distribution (CBD) is a key mechanism for decentralizing RH / FP services at community level ; CHWs are key stakeholders for improving access to LARCs; when they are well trained and supervised, CHWs can adequately provide FP services at the community level; Provision of injectables by CHWs could lead to increased contraceptive use in areas of low contraceptive prevalence, often characterized by high levels of unmet need (BNS), and a lack of access to a wide range of methods and clinical services; good incentives keep CHWs in their role and improves the quality of their services.

\section{Recommendations}

Recommendations made relate to strengthening the development of national policies for the introduction, continuation and extension of contraceptive methods provision at community level, particularly injectables; to strengthening training, supervision and incentives for CHW. Moreover, a continuous supply of medicines and FP commodities and better documentation of experiences and interventions have been suggested.

\section{Study limitation}

The main difficulty for this study was access to documentation. Although several countries have mentioned some task shifting activities, few or no documents could be found. Moreover, the lack of reactivity of stakeholders in getting available documentation also constituted a barrier in some cases.

\section{Conclusion}

Task shifting is already practiced to varying degrees in the countries of the Ouagadougou Partnership. However, documentation could be found mainly for experiences conducted at community level, and particularly with lay health workers. Thus, it appears that, in the West African context, it is possible for well-trained and supported community volunteers to ensure effective and safe administration of hormonal contraceptives. However, the continuation and sustainability of these activities depend on the commitment of governments, partner NGOs and the international community to ensure continuous supply of medicines and FP commodities and technical and financial support to initiatives. Few experiences with health workers or in health facilities could be tracked. Is this by lack of such task shifting experiences or by lack of documentation of these experiences? The question remains. 



\section{Introduction}

Dans le monde, 800 femmes environ meurent chaque jour de causes évitables liées à la grossesse et à l'accouchement. 99 pour cent de tous les décès maternels surviennent dans des pays en développement, dont plus de la moitié en Afrique subsaharienne (OMS, aide-mémoire $n^{\circ} 348$, mai 2014). En outre, la mortalité maternelle est plus élevée en milieu rural et dans les communautés les plus pauvres. En Afrique de l'Ouest, comparativement aux pays anglophones et lusophones, les pays francophones présentent les plus forts taux de mortalité maternelle et infanto-juvénile, les plus forts indices de fécondité, et les plus faibles taux de prévalence contraceptive. Le risque de mourir au cours d'une grossesse ou d'un accouchement y est 3,5 fois plus élevé que dans les autres régions en développement et 270 fois plus élevé que dans les pays avancés (Conférence de Ouagadougou, 2011). Dans cette partie de l'Afrique, il est estimé qu'en moyenne 225 femmes meurent tous les jours en donnant la vie (Partenariat de Ouagadougou, 2011). Le taux de mortalité maternelle varie de 341 décès pour 100000 naissances pour le Burkina Faso (EDS, 2010) à 715 décès pour 100000 naissances vivantes pour la Mauritanie (Rapport MICS IV de 2011). Le taux de mortalité infantile quant à lui varie aussi de 28 décès pour 1000 naissances pour le Burkina Faso (EDS 2010) à 75 décès pour 1000 naissances pour la Mauritanie (MICS IV, 2011). Cette situation est fortement aggravée par l'ampleur des besoins non satisfaits en PF, liée à l'accès limité aux services de santé et à l'information en la matière.

L'indice synthétique de fécondité (ISF) dans les pays francophones de la région reste élevé, avec une moyenne de 5,5 naissances par femme. En plus, 29 pour cent des femmes en âge de procréer ont un besoin non satisfait (BNS) de planification familial, l'un des taux les plus élevés du monde (Partenariat de Ouagadougou, 2014).Le taux de prévalence contraceptive (TPC) y est également faible. II varie de 7 pour cent pour la Guinée (EDS 2012) à 20 pour cent pour le Sénégal (EDS Continue 2014). Le Tableau 1 ci-dessous renseigne sur quelques indicateurs clés des pays francophones de l'Afrique de l'Ouest. 
Tableau 1: Indicateurs clés SR/PF des pays francophones de l'Afrique de l'Ouest.

\begin{tabular}{|c|c|c|c|c|c|}
\hline Pays & $\begin{array}{l}\text { Mortalité Maternelle } \\
\text { (pour } 100000 \\
\text { naissances vivantes) }\end{array}$ & $\begin{array}{c}\text { Mortalité infantile } \\
\text { (pour } 1000 \\
\text { naissances } \\
\text { vivantes) }\end{array}$ & ISF & BNS (\%) & $\begin{array}{l}\text { TPC Méthodes } \\
\text { modernes (\%) }\end{array}$ \\
\hline Bénin & $397^{1}$ & $67^{1}$ & $4,9^{1}$ & $32^{2}$ & $8,7^{3}$ \\
\hline Burkina Faso & $341^{4}$ & $28^{4}$ & $6,0^{4}$ & $23,8^{4}$ & $15^{4}$ \\
\hline Côte d'ivoire & $400^{5}$ & $60,16^{5}$ & $5^{6}$ & $27^{6}$ & $13,9^{6}$ \\
\hline Guinée & $710^{7}$ & $34^{7}$ & $5,1^{7}$ & $21^{8}$ & 77 \\
\hline Mali & $550^{9}$ & $58^{10}$ & $6^{10}$ & $26^{10}$ & $9,9^{10}$ \\
\hline Mauritanie & $715^{11}$ & $75^{11}$ & $4,7^{11}$ & $37,2^{11}$ & $11^{12}$ \\
\hline Niger & $535^{13}$ & $51^{14}$ & $7,6^{14}$ & $19,2^{14}$ & $12^{14}$ \\
\hline Sénégal & $392^{15}$ & $33^{16}$ & $5^{16}$ & $25^{16}$ & $20^{16}$ \\
\hline Togo & $400^{17}$ & $49^{17}$ & $4,8^{17}$ & $38,5^{17}$ & $17^{17}$ \\
\hline \multicolumn{6}{|c|}{ Bénin : ${ }^{1}$ : EDS $2006 ;{ }^{2}$ : EDS 2011-2012 ; ${ }^{3}$ : EDS 2013} \\
\hline \multicolumn{6}{|c|}{ 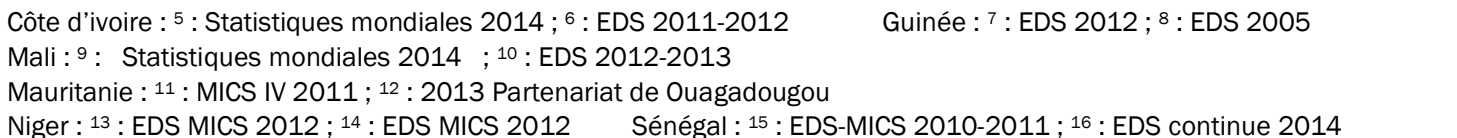 } \\
\hline \multicolumn{6}{|c|}{ Togo : 17 : EDST III 2013; } \\
\hline
\end{tabular}

Le recours à la contraception est bénéfique pour la santé maternelle en empêchant les grossesses non désirées ou trop rapprochées et en évitant les avortements à risque. En répondant à tous les BNS en méthodes modernes de contraception, il serait possible de réduire de 48000 le nombre de décès liés à la grossesse en Afrique sub-saharienne.

Permettre aux femmes de planifier leurs grossesses serait également bénéfique pour la santé de leurs enfants. Une étude menée en 2012 par le Guttmacher Institute dans plusieurs pays en développement a montré que si toutes les naissances étaient espacées d'au moins deux (2) ans, le nombre de décès chez les enfants de moins de cinq (5) ans diminuerait de 13 pour cent. Le nombre diminuerait de 25 pour cent si cet intervalle inter-génésique était de trois (3) ans.

Pourtant, une série de conférences internationales au cours des années 1980-2000 a permis un changement fondamental dans la façon dont le monde perçoit les problèmes de population, et plus spécifiquement de PF. Ainsi:

- Au plan régional, les conférences de Nairobi (1987) et de Niamey (1989) ont marqué le point de départ de l'initiative pour la maternité sans risque, visant la réduction des taux de mortalité maternelle et infantile.

- La déclaration dite de Dakar/Ngor en décembre 1992 a eu pour effet d'harmoniser les positions africaines avant la conférence du Caire de 1994 (voir point suivant).

- La Conférence Internationale sur la Population et le Développement (CIPD) du Caire en 1994 a défini le concept de "santé de la reproduction " et les stratégies de mise en œuvre du plan d'action du même nom. 
- Le Forum régional sur l'enseignement de la santé de la reproduction dans les facultés et les écoles de santé d'Afrique Centrale et Occidentale (Forum SR/ACO), tenu à Ouagadougou en 1996, a permis l'élaboration d'une stratégie commune de formation en SR à travers des plans nationaux de formation.

- La première conférence des pays francophones d'Afrique sur la participation des hommes à la santé de la reproduction, tenu à Ouagadougou en 1998, a permis de situer le rôle de I'homme dans la SR en tant qu'acteur et bénéficiaire.

- Les Objectifs du Millénaire pour le développement (OMD), adoptés en Septembre 2000 lors du Sommet du Millénaire au Siège de l'Organisation des Nations Unies à New York. Les OMD sont au nombre de huit (8), et l'ensemble des 191 États Membres des Nations Unies ont convenu de s'efforcer de les atteindre d'ici 2015. L'OMD 4 est de réduire la mortalité infantile des deux tiers en 2015 par rapport à son niveau de 1990. Pour atteindre cet objectif, il faudra instaurer la couverture universelle par des interventions essentielles, à la fois efficaces et peu coûteuses parmi lesquelles les soins du nouveau-né et de la mère. L'OMD 5 vise à améliorer la santé maternelle, à réduire la mortalité maternelle de 75 pour cent et à rendre l'accès à la médecine procréative universelle en 2015. Mondialement environ 10 pour cent de femmes n'ont toujours pas accès à une contraception sûre et efficace. On estime que la simple satisfaction des besoins non couverts de PF, permettrait de diminuer de près d'un tiers le nombre des décès maternels (OMS, 2014).

Toutes ces conférences ont permis aux pays participants, notamment ceux d'Afrique, de s'accorder sur des questions de SR, y compris la PF, et de leur octroyer une plus grande importance. Plusieurs initiatives ont été développées dans le sens de l'accessibilité et de la promotion de l'espacement des naissances, en encourageant notamment la création ou le changement de politiques en matière de population.

Les initiatives similaires continuent jusqu'à ce jour à mobiliser les pays africains autour de la SR/PF. Ainsi, la Conférence Régionale sur la Population, le Développement et la Planification Familiale tenue à Ouagadougou au Burkina Faso en 2011, a permis la création du "Partenariat de Ouagadougou " qui regroupe les neuf (9) pays francophones de l'Afrique de l'Ouest (Bénin, Burkina Faso, Côte d'Ivoire, Guinée, Mali, Mauritanie, Niger, Sénégal et Togo) et leurs partenaires techniques et financiers. Ce Partenariat vise à accélérer les progrès dans l'utilisation des services de PF dans ces pays. C'est ainsi qu'il s'est fixé pour objectif principal d'atteindre au moins un (1) million de nouvelles utilisatrices de méthodes contraceptives modernes en 2015. Pour cela, il mise sur une meilleure coordination entre les bailleurs de fonds pour optimiser leurs soutiens aux pays, et sur une collaboration et coopération aux niveaux national et régional afin de remédier aux taux élevés des besoins non satisfaits en matière de PF. La déclaration issue de cette conférence de 2011 a spécifié la délégation des tâches comme étant une stratégie prioritaire pour améliorer l'accès aux services de PF (Cf. point $4^{1}$ de la déclaration de Ouagadougou)

La Figure 1 présente la carte des pays du Partenariat de Ouagadougou.

1 Augmenter de $30 \%$ le nombre de professionnels de santé formés et autorisés à offrir la gamme des services de PF et de santé reproductive. 
Figure 1: Carte des pays du Partenariat de Ouagadougou

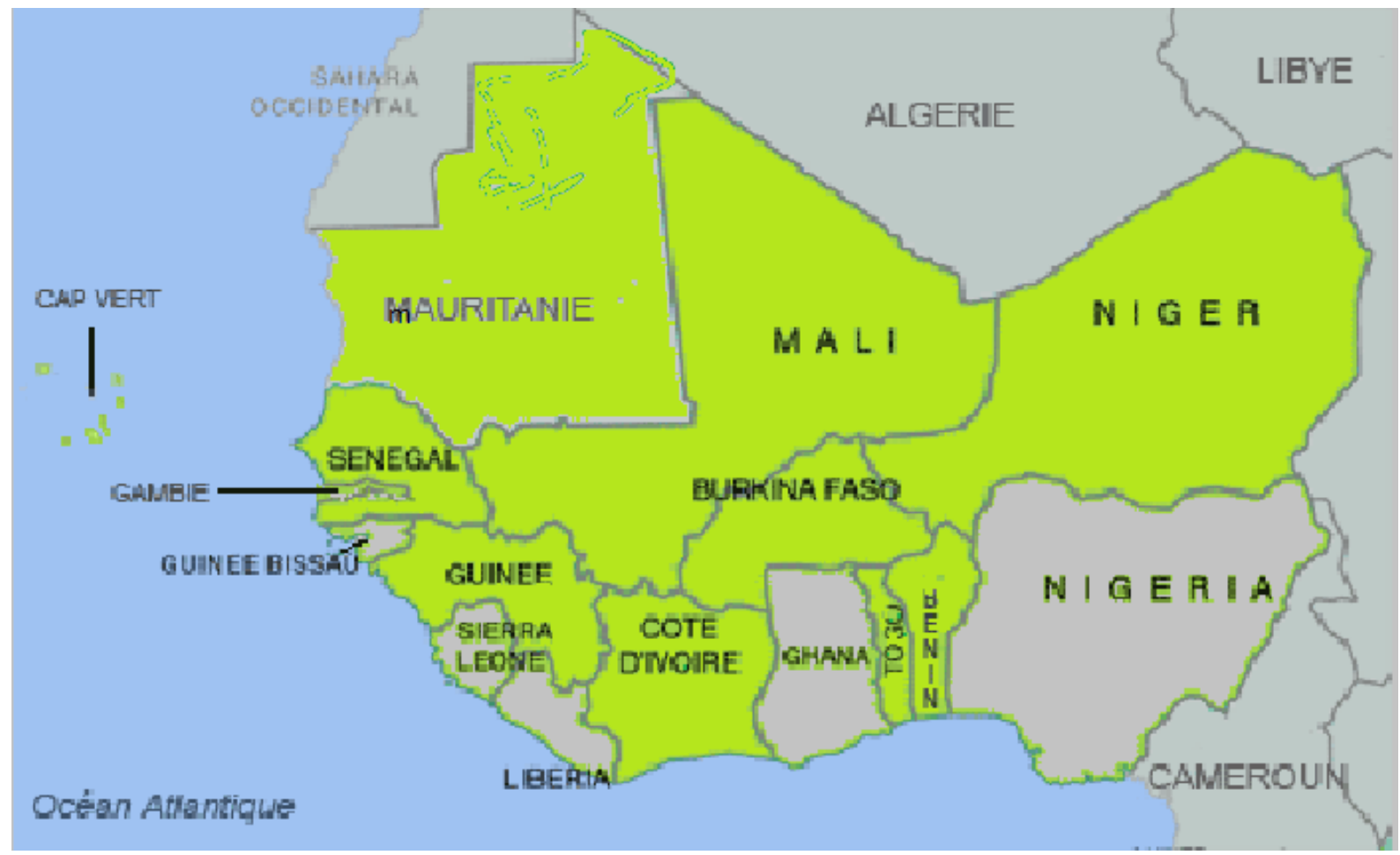

Pays du Partenariat de Ouagadougou

Source : Adapté de http://ekladata.com/wnqXGGuBm4RnYI44HUsqNzMCuTs.gif

En 2012, lors du Sommet de Londres sur la PF, un des objectifs que s'est donné Family Planning 2020 (FP2020) était d'apporter d'ici 2020 la contraception moderne à 120 millions de femmes présentant un BNS dans 69 des pays les plus pauvres du monde. Les pays du PO figurent sur la liste des pays cibles. Le Bénin, la Guinée et la Mauritanie font partie des cinq (5) derniers pays qui se sont engagés en 2013 envers cette initiative (Source : FP2020).

Le Forum Francophone "Sécurité Contraceptive en Afrique Francophone " (SECONAF), a permis, lors de la deuxième réunion annuelle du $\mathrm{PO}$, tenue le 11 novembre 2013 à Addis Abeba, de résumer les convictions et engagements de ses membres en ces termes: "Parler le même langage, agir autrement et ensemble". La rencontre organisée par le Partenariat de Ouagadougou et SECONAF regroupait les délégués des neuf (9) pays du Partenariat.

L'atteinte de l'OMD 5 reste toutefois un défi majeur à quelques mois de l'échéance des OMD. Un défi surtout pour les pays de l'Afrique de l'Ouest francophone qui présentent de faibles niveaux de prévalence contraceptive, avec des taux élevés de BNS en PF et très peu de personnels qualifiés à l'assistance et à l'offre de services de qualité en matière de SR/PF (ONU, 2013).

Enfin, malgré les nombreux défis auxquels ils font face, l'aide aux pays francophones d'Afrique subsaharienne, pour les interventions de PF reste faible. Entre 1997 et 2007, cette aide était estimée à 0,86 dollar américains per capita, comparée à 1,25 dollars américains per capita pour les pays anglophones et lusophones (Partenariat de Ouagadougou, 2011). 


\section{Cadre et Justification de l'étude}

L'OMS a défini 23 agents de santé2 pour 10000 habitants comme le ratio minimum nécessaire pour atteindre les niveaux souhaités de couverture pour les interventions sanitaires essentielles.

Selon l'OMS, il y a un déficit global de personnel de santé de plus de quatre (4) millions de personnes (OMS, 2008). Cette situation est particulièrement grave dans les pays en développement, y compris l'Afrique subsaharienne. En effet, selon le "Rapport sur la santé dans le monde 2006 : Travailler ensemble pour la santé", sur les 57 pays actuellement confrontés à un déficit d'effectifs en personnel de santé, 36 se trouvent en Afrique sub-saharienne. Ce rapport rend aussi compte des inégalités dans la répartition du personnel de santé. Les estimations indiquent que seulement 24 pour cent des médecins et 38 pour cent des infirmières travaillent dans les communautés rurales, alors que la moitié de la population mondiale vit en zone rurale. De plus, les établissements de santé tels que les hôpitaux et dans une moindre mesure les centres et postes de santé, sont le plus souvent concentrées dans les villes et grandes agglomérations, laissant ainsi les zones rurales dépourvues de structures et de personnel de qualité. Dans le même temps, la demande de soins de santé est en augmentation. Pour atteindre les OMD 4 et 5 et le droit à l'accès universel aux services de santé, il faudra se pencher sur cette question cruciale, la pénurie de main-d'œuvre étant un des facteurs clé expliquant l'écart entre l'accès inégal aux services entre zones rurales et urbaines et l'état de santé, et entre riches et pauvres (Foreit \& Raijman. 2011). Un système de santé solide et efficace avec des ressources humaines suffisantes et adéquatement formés, est nécessaire pour faire face à la demande croissante en santé et pour atteindre les objectifs de santé des pays et les engagements internationaux.

$\mathrm{Au}$ cours des dernières années, de nombreuses stratégies ont été expérimentées pour adresser les défis engendrés par le déficit de main-d'œuvre dans le domaine de la santé en vue d'élargir l'accès aux services de PF. La délégation ou le partage des tâches sont quelques-unes de ces nombreuses stratégies.

La délégation qui traduit un transfert des tâches implique la redistribution des tâches. Des tâches spécifiques sont transférées des travailleurs de la santé plus qualifiés aux travailleurs avec moins de formation ou de qualification. Bien que le terme soit souvent utilisé indifféremment pour désigner le partage des tâches ou l'expansion de rôle, le véritable transfert des tâches se produit habituellement dans les milieux cliniques. Par exemple, un bénévole offre une éducation et des conseils aux clients, une tâche déjà effectuée par une infirmière. Cette dernière est ensuite libérée pour effectuer des tâches plus administratives et cliniques.

Le partage des tâches peut se référer au fait que les travailleurs d'un niveau supérieur et ceux d'un niveau inférieur fournissent le même service (par exemple, les travailleurs de niveau inférieur et supérieur fournissent des préservatifs), ou fournissent différentes composantes d'un même service (par exemple, un travailleur de niveau inférieur fournit une éducation sur la PF et renvoie à un travailleur de niveau supérieur qui fournit effectivement des contraceptifs).

Dans cette étude, " transfert des tâches ", " partage des tâches " et " délégation des tâches " sont utilisés de façon interchangeable.

La littérature confirme que les prestataires non qualifiés, avec une formation suffisante, sont en mesure de dispenser toute une gamme de services de santé très utiles, dont des soins d'urgence dans des régions peu desservies (Beaglehole, 2003; Dovlo, 2004; Thairu et Schmidt, 2003; Vaz et al, 1999). De plus, une étude menée en Inde, a montré que les agents de santé communautaires, même ceux dont le niveau

2 Médecins, infirmiers et sages- femmes 
d'alphabétisation est peu élevé, pouvaient fournir efficacement la méthode des jours fixes et la méthode de l'allaitement maternel et de l'aménorrhée (MAMA) (Georgetown University, 2008). D'autre part, d'après les données recueillies par des experts sur 16 projets dans neuf (9) pays, la fourniture de contraceptifs injectables par des agents de santé communautaires qualifiés et soutenue par des programmes était sûre, efficace et acceptée par les clientes (OMS et al, 2010).

L'approche " 3D " (démocratisation, démédicalisation et décentralisation) est un concept introduit au Sénégal et qui fournit un cadre novateur pour la structuration des interventions en PF. La "démocratisation" vise à faciliter l'accès aux services grâce à une approche multisectorielle et participative. La "démédicalisation" a pour objectif de permettre aux travailleurs non-médicaux de fournir une vaste gamme de services en PF aux populations vulnérables (zones rurales, pauvres des zones urbaines, jeunes, déplacés, refugiés) à travers des programmes de DBC. Et enfin, la "décentralisation" vise à renforcer les systèmes de santé aux niveaux de la région, du district et de la communauté tout en améliorant la bonne gouvernance, la responsabilité et la collaboration intersectorielle (Partenariat de Ouagadougou, 2011)

Pour aider les pays à optimiser les performances des agents de santé maternelle et néonatale (SMN), l'OMS a rédigé une série de recommandations notamment pour le partage des tâches dans le domaine de la PF (OMS, 2013). Le Tableau 2 résume les 48 recommandations relatives à la PF.

\section{Tableau 2 :}

Résumé de recommandations des directives Optimiser la SMN pour le partage des tâches dans le domaine de la planification familiale.

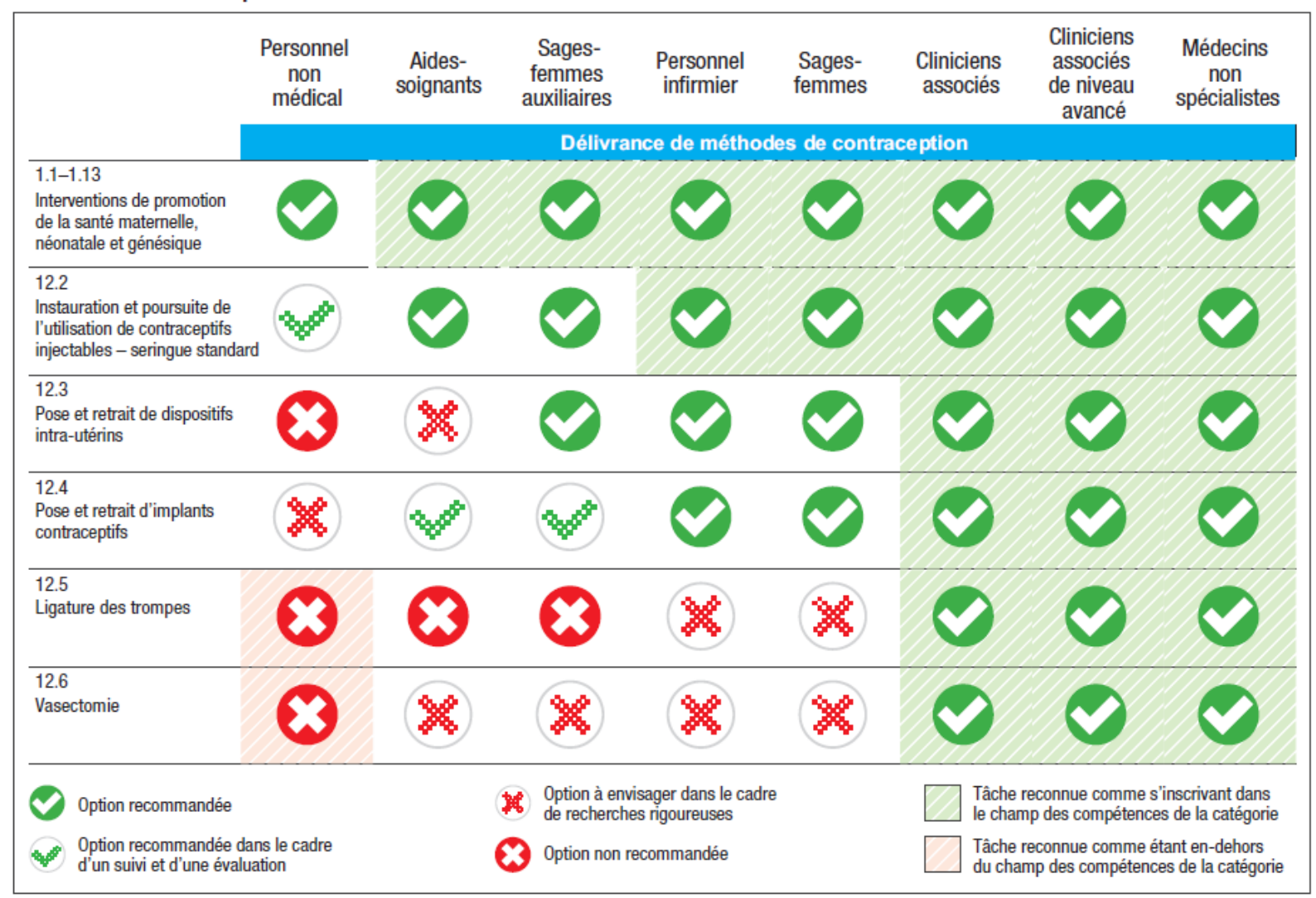

Source : OMS Département Santé et Recherche Génésiques. 2013. La délégation des tâches pour améliorer l'accès aux méthodes de contraception. $p 2$. 
La délégation des tâches semble devenir une intervention porteuse de promesses pour renforcer la couverture sanitaire et l'accessibilité à la contraception. De manière spécifique, l'OMS recommande de déléguer des tâches quand:

- l'accès aux services est limité en raison de la pénurie globale d'agents de santé disposant des compétences pour fournir des méthodes spécifiques ou de leur répartition inégale dans un pays ou une région ;

- il s'avère difficile de fidéliser les catégories de niveau supérieur dans certains milieux comme les zones rurales ;

- les niveaux bas de salaire des agents de santé appartenant aux catégories de niveaux inférieur ou intermédiaire permettent de faire baisser les coûts liés aux services de PF sans compromettre la sécurité des clients ;

- les agents de santé des catégories de niveau supérieur doivent avoir plus de temps libre à consacrer à la fourniture de services exigeant un niveau de compétence technique plus élevé.

Ces agents de santé de niveau inférieur portent différents noms tels que : agents de santé communautaires (ASC), agents de distribution à base communautaire (ASBC), agents de santé villageois (ASV), relais communautaires (RC), aides/auxiliaires de santé communautaire, éducateurs de santé communautaire, etc. Leur niveau d'éducation, leur formation, l'étendue de leurs fonctions et leur statut professionnel varient en fonction des pays et des programmes.

Le faible accès aux services de PF est souligné comme un facteur clé contribuant à des niveaux élevés de BNS (STEP-UP, 2013). Certains aspects des services de PF pourraient être déplacés à des niveaux inférieurs du système de santé et dans les communautés, permettant ainsi l'accès à un large choix de méthodes contraceptives.

Comme de nombreux pays d'Afrique sub-saharienne se sont engagés dans le repositionnement de la PF, il est important de documenter les efforts visant à disposer de main-d'œuvre, pour améliorer l'accès aux services de santé, particulièrement en Afrique de l'Ouest francophone ; et de contribuer à l'échange d'expériences SudSud et à l'apprentissage mutuel. En outre, bien que plusieurs pays aient exprimé leur intérêt pour améliorer les compétences du personnel de niveau inférieur dans l'exécution de tâches cliniques supplémentaires, et pour explorer diverses options de délégation des tâches, des réserves ont parfois été soulevées sur cette stratégie sans que des preuves solides de sécurité et d'efficacité d'une telle réaffectation aient été produites. 


\section{Objectifs}

La présente étude a été menée par le Population Council en collaboration avec le Partenariat de Ouagadougou. Elle a pour objectifs de :

1) Documenter les expériences et leçons apprises dans chaque pays du Partenariat par rapport à la délégation des tâches.

2) Dégager des évidences solides de la sécurité et de l'efficacité de l'approche dans le contexte local et régional.

3) Utiliser les résultats pour une application des connaissances et un plaidoyer au niveau national, régional et international pour aider dans la mise en œuvre de programmes de planification familiale informés par les évidences, y compris les nouvelles technologies telles que l'Anneau Vaginal à Progestérone.

4) Utiliser les résultats de l'étude pour aider au développement de nouvelles approches permettant la prestation de services en dehors des structures de santé par des prestataires de plus bas niveau et l'auto-administration.

\section{Méthodologie}

Cette étude est une revue documentaire visant à fournir des informations clés quantitatives et qualitatives. Elle a examiné et analysé toute la documentation disponible telle que : des études menées dans le cadre de recherches, des rapports réalisés dans le cadre de la gestion des programmes/projets au niveau des zones d'intervention, des documents de politiques élaborés par les institutions étatiques, etc.

Une fiche de collecte standardisée a été développée pour systématiser et harmoniser la collecte des informations disponibles pour chaque pays.

Une recherche sur Internet a été menée à partir des mots-clés suivants: contraception; planification familiale; partage des tâches; délégation des tâches; démédicalisation; transfert des tâches; Partenariat de Ouagadougou; et nom de chaque pays combiné avec les autres mots clés.

De plus, I'Unité de Coordination du Partenariat de Ouagadougou (UCPO) a informé ses points focaux dans les pays pour introduire l'étude. Des contacts de suivi individuel ont ensuite permis de présenter l'étude aux points focaux de manière plus approfondie, d'apporter des précisions sur ce qui était recherché, et de demander leur collaboration dans la localisation des documents et/ou de nous guider, par effet boule de neige, vers d'autres parties prenantes qui pourraient avoir des documents pertinents.

L'analyse a combiné une démarche descriptive, qui a pour objet de décrire et une démarche inductive, qui consiste à partir des informations disponibles pour élaborer une analyse théorique. Cela a permis de présenter un état compréhensif des lieux sur la base des axes thématiques dégagés, dans le but d'apprécier les stratégies définies, leur significativité, et les contraintes dans la mise en œuvre.

Ce rapport est une synthèse des expériences des neuf (9) pays. Les sections sont organisées autour des questions suivantes: Quel environnement politique et réglementaire régit la délégation des tâches dans les pays du Partenariat de Ouagadougou? Quelles sont les différentes expériences de délégation des tâches recensées ? Quelles sont les contraintes à la mise en œuvre de la délégation des tâches ? Quelles leçons peuton tirer de ces différentes activités de délégation des tâches?, et enfin des recommandations et une conclusion sont formulées. Un rapport annexe présente les informations recueillies par pays. 


\section{Environnement Politique, légal et règlementaire}

Des engagements ont été pris par les gouvernements pour l'amélioration de la santé sexuelle et reproductive (SSR) en général et l'offre des services de PF de qualité en particulier. Ainsi, les neuf (9) pays du Partenariat de Ouagadougou ont tous pris l'engagement d'introduire de manière explicite la délégation des tâches dans leurs politiques en matière de SR en général et PF en particulier.

Dans les différents pays, l'environnement politique est généralement favorable à la PF, comme le montrent les différents plans nationaux de développement sanitaire (PNDS), les stratégies nationales de SR, les plans de sécurisation des produits SR, la création de ligne budgétaire pour l'achat des produits contraceptifs pour augmenter l'offre dans les services de PF, l'élargissement des services de la SR aux adolescents, etc. La majorité des pays dispose aussi d'un document de Politiques Normes et Protocoles (PNP) qui sert de ligne directrice opérationnelle dans la prestation des services de santé. II décrit les protocoles et actes d'offre de service que les prestataires de soins doivent observer pour se conformer aux normes. II autorise ou restreint aussi certains services ou actes médicaux à des catégories de prestataires de soins. Ces documents servent de lignes directrices opérationnelles dans la prestation des services de santé au niveau pays.

Plus récemment, les neuf (9) pays du Partenariat ont élaborés des plans d'action nationaux de PF, documents de référence donnant des orientations stratégiques sur le repositionnement de la PF ; le renforcement de la disponibilité des produits, du cadre institutionnel et juridique, et l'accès à des services de PF de qualité.

Le Tableau 3 donne un aperçu de l'état d'avancement des travaux par rapport à ces plans ${ }^{3}$.

3 L'une des actions clefs du Partenariat de Ouagadougou est l'assistance aux pays membres pour le développement de plans d'action spécifiques à la planification familiale. Une approche pour l'affinement de ces plans nationaux basée sur la participation, la collaboration et la décentralisation a été développée et codifiée. 
Tableau 3 : Etat de préparation des plans de repositionnement de la PF dans les pays du Partenariat

\begin{tabular}{|c|c|c|}
\hline Etat d'avancement & Pays & Date de lancement \\
\hline \multirow{8}{*}{$\begin{array}{l}\text { Plan officiellement } \\
\text { lancé }\end{array}$} & Bénin & 29 janvier 2015 \\
\hline & Burkina Faso & 14 juin 2013 \\
\hline & Côte d'ivoire & 13 novembre 2014 \\
\hline & Mali & 3 Septembre 2014 \\
\hline & Mauritanie & 3 septembre 2013 \\
\hline & Niger & 07 février 2013 \\
\hline & Sénégal & $\begin{array}{l}28 \text { novembre } 2012 \\
\text { Atelier de revue du plan le } 5 \text { Février } 2015\end{array}$ \\
\hline & Togo & 13 mai 2014 \\
\hline $\begin{array}{l}\text { Plan affiné mais non } \\
\text { officiellement lancé }\end{array}$ & Guinée & $\begin{array}{l}\text { Plan terminé en septembre } 2013 \\
\text { Non officiellement lancé }\end{array}$ \\
\hline
\end{tabular}

Source : Partenariat de Ouagadougou

La DBC est une forme de délégation des tâches considérée en son temps par certains comme la plus importante innovation en matière de PF (Phillips J. F et al. 1999). Elle a été identifiée comme stratégie d'intervention prioritaire afin d'augmenter le taux de la prévalence contraceptive dans les différents plans d'action des pays du PO.

L'environnement juridique est également propice à la promotion de la PF avec l'existence d'une loi relative à la SSR dans la plupart des pays du Partenariat ${ }^{4}$. Ces lois constituent le cadre général de réglementation. Elles garantissent entre autre les droits des personnes les plus vulnérables et donnent l'accès, sans discrimination aux services de PF.

En ce qui concerne la délégation des tâches, l'exercice de certaines tâches jadis réservées au personnel qualifié est permis par les agents de santé communautaires de façon générale, dans la plupart des pays, à travers des règlements, arrêtés, circulaires ou directives. Cependant, dans la pratique, les tâches dont s'acquittent ces agents de santé dépassent pourtant souvent ce que la loi leur permet de faire. Mais des restrictions sont aussi quelquefois notées dans certains pays avec l'existence de dispositions qui limitent la pratique des agents de santé communautaires et ne leur permettent pas l'offre d'injectables. De manière spécifique pour chaque pays, on peut noter que :

Au Bénin, les RC ne sont autorisés à offrir que les méthodes de barrière (préservatifs, spermicides, méthodes jours fixes (MJF)/ Colliers de Cycle) et à renouveler les contraceptifs oraux. Ces services sont présents dans les modules de formation, mais pas dans le document de politique. Dans les faits, très peu de RC distribuent des contraceptifs oraux ou proposent des services de PF.

En outre, le Bénin a développé une politique de santé communautaire comprenant le C-PIHI (Paquet d'Intervention à Haut Impact Communautaire) qui décrit les services qui doivent être fournis par les ASC et dans quelles circonstances. Les services de PF n'en font actuellement pas partie, mais le Ministère de la

4 La documentation disponible n'a pas permis de renseigner sur l'existence d'une loi SR en Côte d'ivoire, tandis qu'en Mauritanie cette loi est à ce jour toujours à l'état de projet. 
Santé est en train de réviser ses recommandations pour incorporer un paquet normalisé de services PF à fournir au niveau communautaire.

Les Pairs Educateurs (PE) qui opèrent également au niveau communautaire sont assistés par diverses ONG. Ils fournissent les services suivants: Information Education et Communication (IEC), conseils, offre de méthodes (préservatifs et renouvellement de contraceptifs oraux), et orientation/référence (préservatifs (pour les PE qui n'en distribuent pas), contraceptifs oraux (pour les PE qui n'en distribuent pas), DIU, contraceptifs injectables, implants et méthodes permanentes).

De plus, il est envisagé de tester l'administration de contraceptifs injectables au niveau communautaire par les aides-soignants 5 .

Au Burkina Faso, les résultats des interventions liées à l'offre de services à base communautaire (SBC) ont conduit la Direction de la Santé de la Famille (DSF) à développer un curriculum de formation en SBC dont un module est consacré à la DBC des contraceptifs. Le 26 novembre 2008, le gouvernement a adopté le document de politique nationale de contractualisation dans le système de santé. L'approche contractuelle utilise des organisations déjà bien intégrées à la communauté pour la fourniture des services. Elle est mise en œuvre par le Programme d'Appui au Développement Sanitaire (PADS). Le choix de I'Organisation à Base Communautaire d'Exécution (OBCE) qui entre en partenariat avec le district sanitaire suit des critères définis. Un contrat est établit entre le PADS et I'OBCE. L'ECD participe à la formation des membres des OBCE contractualisées et en assure la supervision.

Suite à l'adoption du document de politique nationale de contractualisation, une décision de mettre en œuvre la DBC a été prise en 2009 (pour les produits contraceptifs) à travers la contractualisation avec les ONG de renforcement des capacités (ONG RENCAP).

Des efforts ont été engagés en termes d'innovation, avec par exemple l'introduction du Sayana Press officialisé lors d'une cérémonie de lancement le 10 juillet 2014. Et depuis lors l'expérience est en cours dans 4 régions, à savoir le Centre-Ouest, la Boucle du Mouhoun, les Hauts-Bassins et le Centre et couvrira la période 2014-2016. Cette initiative a été facilitée par l'existence de stratégies avancées et mobiles avec l'accompagnement de partenaires tels que l'ABBEF et Marie Stopes International (MSI). Les sessions de formation à l'endroit des agents de santé ont également permis à ces derniers de peaufiner leur stratégie d'introduction de Sayana Press dans la gamme des contraceptifs de leurs aires sanitaires. Au-delà des stratégies avancées et fixes classiques, certains districts sanitaires ont décidé d'innover. Par exemple dans le district sanitaire de Tougan, c'est une autre stratégie d'introduction et de promotion de Sayana Press qui est expérimentée : le "Djandjoba de la planification familiale " qui est une manifestation annuelle qui mobilise chaque année de nombreuses femmes pour une danse populaire dans les villages de la province du Sourou ${ }^{6}$.

D'autre part, le Ministère de la Santé a signé le 8 novembre 2014 un important accord de collaboration avec le projet Advance Planning Familial de I'ONG Equilibres et Populations (EquiPop) pour l'expérimentation de la délégation des tâches dans les régions du centre et de la Boucle du Mouhoun 7 . Cette délégation des tâches

5 Parmi les auxiliaires de santé (aides-soignants, secouristes et matrones), seuls les aides-soignants sont retenus dans le document des normes comme personnel capable d'assister les Infirmiers et les Sages-Femmes dans les formations sanitaires. L'aide-soignant est la personne formée pour aider l'infirmier ou la sage-femme dans ses tâches quotidiennes en matière de soins et de prévention.

6 Au cours de ces manifestations, les messages sur la planification sont diffusés et de nombreuses femmes trouvent l'occasion d'adopter en toute discrétion des méthodes de PF.

7 Avec le soutien de l'AFP et Futures Group, en collaboration avec le MS, EquiPop a organisé une visite du site pour les représentants de la DSF et la société civile au Togo, afin d'apprendre de leur expérience par rapport à l'utilisation des agents de santé communautaires pour la première prescription de la pilule et l'offre d'injectables. Les résultats positifs du Togo ont conduit le 
autorisera les accoucheuses brevetées et les infirmiers (ères) à administrer les méthodes de contraception réversibles de longue durée, et les ASC à prescrire la première pilule. L'accord de collaboration a rencontré l'approbation des bailleurs de fonds du secteur de la PF qu'Equipop cherche à mobiliser. Cet accord est le résultat d'un plaidoyer coordonné par le Groupe de travail technique de la Santé Reproductive (GT/SR) dont Equipop est membre. Celui-ci continue le plaidoyer afin de prévenir et débloquer les contraintes politiques et financière, et donner au secteur public le rôle de supervision dans l'expérimentation du projet pilote. EquiPop prévoit de coordonner le projet pilote sur une période de deux ans (2015-2016).

En Côte d'ivoire, la situation de crise a désorganisé tout le système d'information sanitaire. En dehors des données issues des activités des organisations humanitaires, depuis le déclenchement de la crise postélectorale de 2002, très peu d'études d'envergure nationale ont été menées. Mais dans l'optique d'accélérer l'atteinte de l'OMD 5, les interventions clés suivantes alignées sur les objectifs des documents cadres nationaux, et qui sous-entendent des activités de délégations des tâches, ont pu être documentées :

- Extension de I'offre de services de PF clinique et de DBC des produits contraceptifs dans 800 structures de santé publiques et privées, particulièrement en milieu rural et périurbain, pour les femmes de 15 à 49 ans ;

- Promotion de l'utilisation des services de santé maternelle, en particulier la PF, auprès des femmes et des hommes notamment en milieu rural et périurbain.

Par ailleurs, la Direction de la Santé Communautaire et de la Médecine de Proximité (DSCMP) a validé le cadre conceptuel des services de santé à base communautaire en 2011.

En Guinée, plusieurs documents clés matérialisent des options fortes des autorités nationales pour développer des interventions communautaires de PF :

En 1996, l'Association Guinéenne pour le Bien-être familial (AGBEF) a élaboré un premier document de Stratégie Nationale des Services à Base Communautaire (SBC) dans le but d'obtenir un cadre de référence pour les activités communautaires. Depuis, plusieurs expériences pilotes de stratégies à base communautaire ont été mises en œuvre avec l'appui d'ONG et associations.

De 2000 à 2012, la stratégie de la Prise en Charge Intégrée des Maladies du Nouveau-né et de l'Enfant (PCIMNE) et la PF ont été intégrées dans un paquet commun pour faciliter au niveau national l'accès des femmes aux services de PF en même temps qu'elles utilisent les services de PCIMNE.

En 2012, la Guinée a élaboré un document de "Politique Nationale de Santé Communautaire" pour servir de référence aux acteurs et interventions. Au-delà de l'insuffisance numérique et qualitative des ressources humaines en santé pour l'offre de PF et des méthodes de longue durée en particulier, le constat est que les financements alloués au secteur de la santé ne permettent pas d'ajuster de façon significative les personnels qualifiés liés à la pratique de sage-femme et d'assurer la performance du système de soins. Face à ces difficultés, le Ministère de la Santé et de l'Hygiène Publique (MSHP) a pris la décision de déléguer certaines tâches aux auxiliaires de santé pour améliorer l'accès aux services pour les communautés rurales. C'est ainsi que les activités liées au DIU ont été déléguées aux accoucheuses, et le Depo-Provera aux autres ASC.

Au Mali, l'autorisation est faite aux RC d'offrir les méthodes de barrière et les pilules en première prescription dès les années 1990. L'autorisation est aussi faite aux accoucheuses traditionnelles de fournir des services de PF dans les villages grâce à une directive émise en 2005.

En 2009, la Direction Nationale de la Santé (DNS) et ses partenaires ont élaboré une nouvelle stratégie pour la DBC, appelée "Soins essentiels dans la communauté ", qui a été lancée en 2011. Cette stratégie a été mise

Directeur de la Santé Familiale à soutenir le protocole d'entente pour la délégation des tâches au Burkina Faso. Plusieurs ateliers ont été organisés entre EquiPop et le Ministère de la Santé pour la modification et la validation de ce protocole. 
en place dans un contexte où 40 pour cent de la population vivent au-delà de $5 \mathrm{kms}$ d'un centre de santé communautaire (CSCOM). II était nécessaire de décentraliser l'accès aux soins en créant un nouveau palier dans la pyramide sanitaire, l'Agent de Santé Communautaire (ASC), afin de rapprocher populations et soins de santé de qualité. Le paquet de soins mis en œuvre par l'ASC, en collaboration avec les relais et les Accoucheuses Traditionnelles Recyclées comprend, entre autres, la PF et la communication pour le changement social et de comportement.

L'intégration en 2012 de la DBC, et plus particulièrement celle des injectables, dans le plan de repositionnement de la PF comme objectif spécifique, a permis de franchir une nouvelle étape importante, de même que l'inclusion dans les PNP de l'insertion des implants par les matrones la même année.

En Mauritanie, la DBC des préservatifs et des pilules de réapprovisionnement est effective depuis le début des années 2000, mais uniquement avec l'appui des ONG et associations. Cette situation perdure jusqu'à présent, malgré l'élaboration en 2013 d'un document de politique de délégation des tâches appelé "Stratégie Nationale de Délégation des tâches ". L'accès des populations rurales à la PF est difficile. Non seulement les initiatives pour les atteindre sont insuffisantes, mais les leaders religieux ne sont pas assez impliqués dans ce domaine. La loi sur la SR n'est toujours pas adoptée et certains textes réglementaires restreignent les services à base communautaire. En effet jusqu'en 2011, pour une première consultation et prescription, les femmes devaient consulter un médecin, un infirmier ou une sage-femme exerçant dans une formation sanitaire (hôpital, centre de santé ou poste de santé). Et jusqu'en 2014, l'offre de méthodes de longue durée était limitée aux médecins et sages-femmes.

Au Niger, la mise en œuvre de la délégation des tâches a été facilitée par l'Arrêté Nº31 /MSP/MDS/P/PF qui autorise depuis le 30 juillet 1992 les agents de santé villageois (ASV) à offrir certaines prestations en PF. II leur est permis la distribution des moyens contraceptifs de barrière (préservatifs, spermicides) et le réapprovisionnement des contraceptifs oraux. Par ailleurs, un plan d'harmonisation des stratégies d'intervention à base communautaire est en voie d'adoption. Mais déjà, des mesures ont été prises telles que l'accroissement de l'offre de santé, surtout en milieu rural, à travers l'élargissement du réseau de cases de santé, la mise en œuvre d'approches à base communautaire, et la promotion de la DBC des contraceptifs par la création de sites DBC.

La révision de l'environnement politique est en cours et de nouveaux textes PF sur les conditions de dispensation sont en cours de validation. Ces nouvelles dispositions devraient permettre aux agents de santé qualifiés d'offrir les injectables intramusculaires (IM) et sous cutanés (SC) et aux agents distributeurs d'offrir les injectables sous cutanés (Sayana Press) ${ }^{8}$ puisque le Ministère de la Santé a donné à l'Association Nigérienne de Marketing Social (ANIMAS) le mandat de développer le Sayana Press dans le réseau pharmaceutique national et dans 100 villages DBC de la région de Maradi.

Au Sénégal, le gouvernement, à travers l'adoption du Plan National de Développement Sanitaire (PNDS) a opté dès les années 1990 pour une politique de décentralisation sanitaire avec l'accent sur l'appui aux initiatives communautaires pour faire face au déficit de ressources humaines et améliorer l'accès aux services pour les populations rurales.

En 2008, le Ministère de la Santé a soutenu une initiative pilote permettant aux matrones et ASC des cases de santé de fournir des pilules contraceptives. En 2012, le Ministère a autorisé les matrones et ASC à offrir les

8 Le Sayana press a été introduit au Niger en Juillet 2014 
méthodes injectables au titre d'études pilotes et a soutenu l'introduction du Depo-subQ Provera 104 TM en Uniject (renommé depuis lors "Sayana press").

Pour rendre opérationnelle la volonté du gouvernement de rapprocher les prestations de services des populations, le Sénégal a élaboré une Politique Nationale de Santé Communautaire en septembre 2013. L'approche stratégique principale comprend la mise en place des dispositions de l'approche " 3D " à travers la mise en œuvre des interventions de santé communautaire.

Le 11 août 2014 le Ministère autorise à travers la circulaire 008938 MSAS/DGS/CSC l'offre initiale de contraceptifs injectables sous forme intramusculaire (Depo provera) et sous-cutanée (Sayana Press) au niveau communautaire dans tous les districts sanitaires et conformément au cadre de passage à l'échelle des expériences communautaires réussies.

Au Togo : la mise en place de stratégies nationales d'intervention à base communautaire s'est faite à travers la distribution de produits contraceptifs, y compris l'injectable, avec l'appui d'ONG et d'associations (contractualisation). Le Ministère de la Santé a signé en 2011 un Protocole d'Accord habilitant les ASC à administrer les contraceptifs hormonaux oraux et injectables ${ }^{9}$.Avant 2011 , pour une première consultation et prescription, les femmes devaient consulter un personnel de santé travaillant dans un centre santé. Depuis octobre 2011, exception est faite dans les districts de Blitta, Haho, Tchamba, Kpendjal et Vo où les ASC offrent à titre expérimental la première prescription des méthodes contraceptives de courte durée d'action. Et cela avant même que la Politique Nationale des interventions à base communautaire ne soit révisée.

Au cours de la même année, le MS a pris l'initiative de tester le modèle de délégation des tâches à travers la mise en œuvre du paquet intégré de PF et du projet " Prise en Charge Intégrée des Maladies du Nouveau-né et de l'Enfant au niveau Communautaire (PCIMNE-C) ", dans les districts pilotes de Haho et Blitta,

En novembre 2014, Opportunity Fund a subventionné I'Association Togolaise pour le Bien Etre Familial (ATBEF) pour un objectif de plaidoyer consistant à amener au plus tard en mai 2015, le Ministère de la Santé à réviser la politique nationale de 2013 concernant les interventions communautaires pour introduire une nouvelle disposition autorisant la fourniture des injectables et de la première prescription de pilule par les ASC.

Ainsi, dans l'ensemble des pays du partenariat, il apparait que les environnements politiques, légaux et règlementaires sont assez favorables à la délégation de tâches. Exception faite peut-être pour la Mauritanie où il est noté une volonté politique de la part des décideurs qui n'est pas assez forte, et une absence de leadership du gouvernement dans à la mise en œuvre de la stratégie nationale de la délégation des tâches. Ce qui, par ailleurs, pourrait constituer un frein pour la mise en œuvre réussie de son plan de repositionnement de la PF.

9 Le partage des tâches pour les injectables ne faisait pas partie de la politique nationale sur les interventions au niveau communautaire. Leur introduction par le projet AWARE II en 2011 a été autorisée par un mémorandum d'accord avec le Ministère de la Santé. 


\section{Expériences de délégation des tâches par pays}

Les expériences de délégation de tâches recensées se sont déroulées le plus souvent en milieu rural bien que quelques-unes se soient déroulées en milieu urbain en Côte d'Ivoire et en Guinée. Les sections suivantes résument les informations recueillies pour chaque pays, et de manière globale, les difficultés rencontrées dans le cadre de la mise en œuvre des projets.

\section{BENIN}

Même si des projets de délégation des tâches sont mentionnés à travers la littérature, le déficit de documentation relatif à ces expériences n'a permis de fournir que quelques informations sur le projet intitulé Programme Survie de l'Enfant dans l'Ouémé (PSEO), mené de 1997 à 2001. Sur financement de I'USAID, I'ONG Africare a développé dans la commune de Pobè des activités à base communautaire, parmi lesquelles la promotion de la PF et la lutte contre les IST/SIDA. Au total, 54 ASBC ont été sélectionnés par la communauté au cours d'ateliers villageois, et formés pour mener, entre autres, les tâches d'éducation/sensibilisation en PF et de DBC de spermicides et préservatifs.

\section{BURKINA FASO}

Au Burkina Faso, la DBC a commencé en 1995 dans trois (3) régions (Sud-ouest, Boucle du Mouhoun et Est) à travers le Projet Planification Familiale appuyé par la GIZ (ex GTZ, Coopération allemande au Développement). La DBC concerne les produits non prescriptifs (préservatifs, méthode du collier, spermicides) et prescriptibles $(\text { pilule })^{10}$ à travers l'expérimentation de deux (2) approches:

- Approche classique

La distribution à base communautaire est entièrement organisée et prise en main par les structures sanitaires. Les services de santé confient les activités de DBC aux ASBC, sous la supervision des équipes de santé de base. Les prestataires de santé effectuent la formation, la supervision et le suivi des ASBC.

- Approche contractuelle

La contractualisation des SBC est mise en œuvre au niveau périphérique dans les 13 régions sanitaires du pays, à travers 18 ONG RENCAP. 160 OBCE sont mises à contribution pour mener cette approche. En 2014, 61 districts sur 63 (soit 94 pour cent) et 1125 formations sanitaires ont été couverts par la contractualisation. En même temps que la DBC, des activités d'Information Education et Communication/Communication pour le changement de comportement (IEC/CCC) sont menées sur les produits.

10 A noter que la femme doit recevoir au préalable une prescription de la structure sanitaire. L'agent DBC n'assure que le réapprovisionnement. 
La contractualisation des services de santé au Burkina a permis une grande implication des différents acteurs : l'Etat, la société civile, les communautés et les partenaires techniques et financiers. Les exemples de délégation des tâches expérimentées dans le cadre de cette approche et qui ont pu être identifiées sont présentés ci-dessous.

\section{Projet PRAJA/SR}

Ce projet a été coordonné durant 4 ans (de 2010 à 2014) par le Conseil Burkinabé des Organisations de Développement Communautaire (BURCASO) ${ }^{11}$ et mis en œuvre par des associations locales au profit des vendeuses ambulantes, des travailleuses du sexe, des femmes des marchés et yaars ${ }^{12}$. II s'est déroulé dans les villes de Niangoloko, Bobo Dioulasso, Ouagadougou, et Pouytenga grâce aux appuis techniques et financiers du PADS et de l'UNFPA.

Le projet avait pour objectif de contribuer à réduire les BNS en PF chez ces cibles au niveau des villes carrefour situées sur l'axe routier international Niangoloko-Cinkansé, à travers la sensibilisation sur la PF, la DBC de contraceptifs (condoms masculins et féminins, pilules, colliers), la référence et le suivi des références auprès des formations sanitaires, et le suivi/supervision des activités.

En décembre 2013, au bout d'un an d'intervention, le projet a permis de réaliser 10205 séances de sensibilisation en PF et 1205 références, et de distribuer 88305 condoms masculins, 152 condoms féminins, 1047 plaquettes de pilules, et 13 colliers.

\section{Projet de distribution à base communautaire des produits contraceptifs non prescriptibles par des pairs éducateurs}

Le Réseau Africain Jeunesse Santé et Développement dans sa stratégie de promotion de la santé sexuelle/santé de la reproduction des adolescent(e)s et des jeunes au Burkina Faso a initié un projet de DBC des produits contraceptifs non prescriptibles. Ce projet couvrait 20 provinces pour sa phase pilote. Financé par I'UNFPA à travers le PADS, deux (2) sessions de formation au profit de 40 pairs éducateurs se sont tenues respectivement à Bobo-Dioulasso et Ouagadougou en octobre 2010.

Avec la DBC, les PE ont dans leurs kits de sensibilisation des préservatifs masculins et féminins, des pilules et des colliers, destinés à être vendus à leurs camarades. La formation a consisté à donner aux PE des connaissances sur les pairs, la SR, les grossesses non désirées et les méthodes de contraception qui leur étaient fournies, le VIH/SIDA, ses modes de transmission et ses méthodes de prévention et les techniques de communication telles que le counseling et la causerie éducative. Ces pairs éducateurs servent d'agents communautaires auprès de leurs camarades. En cas de difficultés sur le terrain ils doivent référer leurs clients au centre de santé. Ils sont autorisés à vendre leurs produits à leurs pairs seulement après prescription par un agent de santé. La phase pilote du projet a débuté en novembre 2010.

\section{Programme Santé Sexuelle, Droits Humains (PROSAD)}

Le Programme Santé Sexuelle, Droits Humains (PROSAD) de la GIZ a été mis en place en janvier 2004 dans le but d'adapter les services de PF aux besoins des usagers dans les centres de santé en renforçant les capacités des travailleurs de ce secteur. La GIZ, à travers ce programme, apporte un soutien à la formation et à la supervision des agents de santé ainsi qu'au personnel d'organisations non gouvernementales et communautaires dans le cadre de la DBC de contraceptifs (pilule et préservatifs masculins et féminins). La GIZ offre également des formations en IEC/CCC sur la PF et le VIH/SIDA dans les régions de l'Est et du Sud-Ouest et à Ouagadougou. Le programme produit des films et des supports multimédia et éducatifs dans plusieurs langues nationales et locales sur les manières d'aborder la PF avec les familles. PROSAD suit et évalue les

11 Cette structure est le répondant pays de l'ONG Pathfinder International dans le cadre de ce projet.

12 Lieu dédié à la recherche de l'or. Dans l'une des langues du Burkina Faso, le Mooré, ce mot signifie le 'marché' et désigne le centre de la mine où les transactions se font. 
activités des organisations formées par la GIZ pour offrir des services de PF, y compris celles impliquées dans le programme de distribution communautaire de contraceptifs. PROSAD devrait prendre fin en 2015.

\section{Projet Promotion des comportements à moindre risque en santé de la reproduction}

Ce projet, mis en œuvre par Family Care International ( $\mathrm{FCl}$ ), fait suite au projet d'appui au "Programme national de lutte contre les fistules obstétricales pour l'amélioration de la sécurité et du bien-être des populations du Sahel 2009-2012 " qui comprenait une composante PF et pour laquelle FCl s'était vue confier le volet communautaire.

$\mathrm{FCl}$ mène des campagnes de plaidoyer dans le but d'encourager le soutien national et communautaire à la santé reproductive, tout en apportant un appui technique aux institutions gouvernementales, ONG et organisations communautaires. FCl est membre de ONG RENCAP et dirige 10 organisations à base communautaire. $\mathrm{FCl}$ apporte aussi un appui technique aux districts sanitaires de Dori, Djibo, Gorom et Sebba dans la région du Sahel pour la mise en œuvre d'une DBC de contraceptifs, utilisant des relais communautaires.

Par ailleurs, une initiative a été développée avec $\mathrm{FCl}$ dans les zones de Barsalogho et du Sahel pour assurer le réapprovisionnement à domicile de la pilule, en toute confidentialité. Cette approche de DBC a la particularité de réduire potentiellement la stigmatisation dans la mesure où elle est intégrée à d'autres services. Les bénéficiaires potentielles sont identifiées à l'aide d'un registre géré par les animateurs de l'ONG, ce qui permet de les localiser et de les réapprovisionner à domicile en contraceptifs.

En moins d'un an d'exécution de la DBC sur le terrain, les activités de communication réalisées se chiffraient à 10061 causeries, 74404 entretiens individualisés, 6791 visites à domicile (VAD), 885 théâtres-fora et 1570 projections de films. 336615 condoms masculins, 2711 condoms féminins, 338 colliers et 50507 pilules ont été distribués. Les ASC ont référé 21118 femmes pour la PF dans les formations sanitaires.

\section{Projet de plaidoyer dans le cadre de l'initiative Advance family planning}

Dans sa volonté d'accompagner le gouvernement du Burkina Faso dans ses engagements, Equilibres \& Populations dans le cadre de l'intiative Advance Family Planning met actuellement en œuvre un projet qui se veut de contribuer à augmenter l'investissement financier, surtout auprès des mairies ${ }^{13}$ et l'engagement politique nécessaires pour favoriser l'accès pour tous à la planification familiale volontaire de qualité, à travers des techniques de plaidoyer fondées sur des résultats de recherche pour de meilleures politiques autour de la délégation des tâches.

\section{COTE D'IVOIRE}

Projet "Les services intégrés de santé sexuelle et de la reproduction de qualité sont disponibles pour répondre aux besoins des populations"

C'est la recrudescence des violences sexuelles dans les quartiers précaires d'Abidjan lors de la crise postélectorale de 2002 et la difficulté pour un grand nombre de femmes d'obtenir les contraceptifs (surtout hormonaux), distribués uniquement par les services cliniques de l'ONG Association de Soutien à I'Autopromotion Sanitaire Urbain (ASAPSU), qui ont principalement motivé la mise en œuvre de ce projet.

13 La Commune de Bobo Dioulasso a adopté sa première allocation budgétaire pour les services de planification familiale, le 10 Mars 2015. L'attribution de 1.000.000 FCFA (2.000 USD) sera incluse dans le Plan Communal de Développement de Bobo Dioulasso 2014-2015. 
En accord avec le Programme National de la Santé de la Reproduction, le projet a été mis en œuvre en 2011, à Abobo, Attecoube, Marcory et Yopougon en faveur des couples et des femmes en âge de reproduction. En Juillet 2011, I'ASAPSU a organisé une session de formation en DBC de PF de 19 animatrices communautaires et un médecin chargé du suivi et évaluation. Les principales activités du projet étaient la sensibilisation de proximité, la sensibilisation de masse, I'IEC (groupes de paroles), la dispensation des méthodes contraceptives non prescriptibles et le réapprovisionnement en pilules, et la référence des cas de violences sexuelles rencontrées dans les quartiers.

Les activités des ASC sur le terrain ont permis de recruter 252 nouvelles utilisatrices, et 24583 personnes ont été touchées au cours des séances de sensibilisation. 11580 pilules et 285 injectables ont été vendus, 1 741 préservatifs masculins et 312 préservatifs féminins distribués.

En dehors de ce projet, quelques activités s'inscrivant dans le cadre de la délégation des tâches en Côte d'Ivoire ont pu être identifiées, mais une documentation suffisante n'a pu être recueillie. Il s'agit de :

\section{Formation de relais communautaires en techniques de CCC}

Le Programme National - PNSR/PF - avec l'appui de I'OMS, a organisé en août 2012 des sessions de renforcement des capacités de 50 relais communautaires des districts sanitaires de Jacqueville, Dabou, Aboisso, Gagnoa et Divo sur les techniques de CCC et sur la PF.

\section{Activités de la Direction de la Santé Communautaire et de la Médecine de Proximité (DSCMP)}

Les activités suivantes ont été réalisées par la DSCMP en 2011:

- Adaptation et validation des modules de formation des formateurs et des ASC pour la DBC des produits contraceptifs ;

- Formation de 69 formateurs des ASC du district de Toumodi pour la DBC ;

- Supervision de la formation de 60 ASC du district de Toumodi pour la DBC.

\section{GUINEE}

Quelques expériences de délégations de tâches ont pu être identifiées.

\section{Projet pilote de distribution communautaire des préservatifs}

En 1995, le Ministère de la Santé, en collaboration avec l'AGBEF, a lancé en Haute Guinée le premier projet pilote de distribution communautaire de préservatifs avec 50 agents communautaires. Cependant la documentation disponible n'a pas permis d'en savoir davantage sur cette initiative.

\section{Projet de santé reproductive et sexuelle communautaire durable en région forestière de la Guinée}

En 2006, l'AGBEF a mis en œuvre une intervention de 3,5 années pour augmenter la connaissance et I'utilisation de la PF dans cinq (5) préfectures de la région forestière. Les approches utilisées comprenaient le recrutement et la formation d'agents communautaires pour offrir des informations (communication de masse), distribuer des méthodes contraceptives modernes, et orienter les populations dans la zone du projet.

Le grand nombre de grossesses chez les adolescentes dans les écoles secondaires des 5 préfectures a également conduit le projet à mettre en place un volet sur la santé sexuelle et les besoins en PF des jeunes, sachant que l'activité sexuelle précoce combinée à une faible utilisation de la PF conduit souvent à des grossesses non désirées menant à l'abandon de l'école et/ou à un avortement dangereux. De plus, selon l'EDS 2005, plus de 33 pour cent des jeunes filles de 15 à 19 ans ont eu un enfant ou sont tombées enceintes. Les 
principales activités du volet du projet destiné aux jeunes étaient le counseling, la distribution de contraceptifs modernes par les professeurs et élèves, et la référence vers les ASC.

Les données ont montré que le nombre de personnes de tout âge qui a adopté une méthode moderne a considérablement augmenté au cours du projet. Ainsi, le nombre d'utilisatrices est passé de 11000 à près de 100000 utilisatrices dans la zone du projet. De plus, le pourcentage de filles scolarisées ayant expérimenté une grossesse a baissé de 9 pour cent à un peu moins de 3 pour cent en deux (2) ans.

\section{Projet PRISM II (Pour Renforcer les Interventions en santé de la reproduction et MST/SIDA)}

Ce projet est une initiative de la République de Guinée dans le cadre de sa coopération bilatérale avec l'USAID. Il a été mis en œuvre par Management Sciences for Health (MSH) de 2003 à 2007. Les zones d'intervention du projet correspondent à la région naturelle de la Haute-Guinée ainsi que la préfecture de Kissidougou, couvrant ainsi toutes les neuf (9) préfectures des régions administratives de Kankan et Faranah.

Le rapport des activités et résultats de PRISM II pour le trimestre du 1 1er janvier au 31 Mars 2006 montrent que le projet a formé 725 agents communautaires dans la prescription et la distribution de contraceptifs oraux et de vitamine A, portant le nombre total d'agents formés en Haute Guinée à 1507 . Parmi la dernière vague d'agents communautaires formés, 59 pour cent étaient des femmes, confirmant la tendance "féminisation" du programme. En Mars 2006, tous les villages ciblés à Kankan, Kérouané et Faranah ont été couverts par PRISM seul14. Les activités des agents de la DBC ont montré une augmentation remarquable du nombre de nouveaux clients dans toutes les préfectures.

\section{Initiative Onchocercose - Santé de la Reproduction/Guinée (2006-2012)}

Ce projet a été mis en œuvre par AGBEF/Helen Keller Internationale (HKI) sous financement de la Banque Mondiale dans les zones du programme Onchocercose, avec 4300 agents communautaires (AC) ou distributeurs communautaires (DC). Il avait pour objectif général de contribuer à l'intégration de la SR dans la stratégie du Traitement par l'Ivermectine sous directives communautaires (TIDC) en vue d'améliorer l'accès aux services et produits de SR au niveau communautaire dans les zones d'intervention spéciale.

Les résultats de l'évaluation interne des activités montrent qu'en 2007 les AC ont distribué 3025 pilules et 965403 préservatifs. Et en 2011, les AC et DC ont distribué 34584 pilules et 5054757 condoms. En 2011, les DC ont référées 10 femmes pour le DIU, et 1218 pour les injectables.

\section{Développement des services de PF à base communautaire dans 43 Sous-Préfectures et 4 Communes urbaines SONU/MURIGA (Mutuelle des Risques liés à la Grossesse et à l'Accouchement) de 2009 à 2011, en Basse et en Moyenne Guinée.}

Bien que des traces de ce projet financé par I'UNFPA aient été retrouvées, le manque de documentation détaillée n'a pas permis d'en savoir suffisamment.

14 Dans certaines zones, la mise en œuvre des activités s'est effectuée en partenariat avec d'autres structures telles que l'Agence Adventiste d'Aide et de Développement (ADRA) pour tous les villages de Siguiri, Save the Children pour les zones de Kouroussa et Mandiana, et Africare pour les villages de Dabola et Dinguiraye. 


\section{Projet pilote d'introduction du Depo-Provera dans le paquet d'activités des agents de santé communautaire}

En 2012, un projet pilote d'introduction du Depo-Provera dans le paquet d'activités des ASC a été mis en œuvre dans la préfecture de Mandiana par le Programme Intégré de Santé Maternelle et Infantile (MCHIP)/JHPIEGO, sous financement de I'USAID, en collaboration avec la DRS de Kankan et la Direction Préfectorale de la Santé (DPS) de Mandiana.

Lors de la première phase (juillet-décembre 2012), 283 nouvelles utilisatrices et 152 utilisatrices régulières ont obtenu l'injectable auprès d'un ASC. L'évaluation faite pendant cette phase a montré que sur les 29 utilisatrices interviewées, toutes ont dit qu'elles recommanderaient les services de l'ASC à une amie, et qu'elles comptaient revenir chez l'ASC pour leur prochaine injection. Parmi les utilisatrices interviewées, 20 (69 pour cent) ont souffert d'effets secondaires mineurs dont 18 (90 pour cent) se sont rendues chez l'ASC pour la prise en charge. 2 (10 pour cent) n'ont rien fait et ont attendu que les effets secondaires s'améliorent. Malgré la survenue de ces effets secondaires mineurs chez les utilisatrices, aucun cas d'abandon n'a été signalé.

Quant à la deuxième phase (janvier à juin 2013), 519 nouvelles utilisatrices et 359 utilisatrices régulières ont obtenu l'injectable auprès d'un ASC. Suite à ces résultats, la DPS de Mandiana a adressé un plaidoyer au Ministère de la santé pour l'extension du projet dans d'autres préfectures de Kankan. Une proposition d'élaboration d'un document pour la mise à l'échelle nationale de la DBC de l'injectable a été avancée afin de faciliter et guider l'extension de l'injectable au niveau communautaire. Le statut actuel de ce document n'a pu être connu au moment de la présente étude.

\section{MALI}

Le Mali a été l'un des pays pionniers en Afrique de l'Ouest francophone pour l'introduction d' un programme de DBC des contraceptifs. En Juillet 1990, le Ministère de la Santé et de l'Hygiène Publique (MSHP) a décidé d'expérimenter un projet de DBC de contraceptifs (préservatifs et spermicides). Ce projet a été exécuté par la Division Santé Familiale et Communautaire (DSFC) sous forme de recherches opérationnelles dans deux régions (Koulikoro et Sikasso) sur financement de I'USAID avec l'appui technique du Population Council. Le projet a formé et déployé des agents communautaires qui ont distribué des préservatifs et des spermicides dans les zones rurales. En 1994, le projet pilote a introduit les contraceptifs oraux et étendu le programme de la DBC dans plusieurs régions.

Au terme de trois années et demie d'expérimentation, les études ont démontré que non seulement l'approche DBC était techniquement faisable et culturellement acceptable, mais aussi qu'elle avait contribué de façon statistiquement significative à l'augmentation du niveau de connaissance et du taux d'utilisation des méthodes modernes de PF par la population cible. Ainsi, le MSHP a demandé et obtenu de I'USAID/Bamako, le financement de l'extension des activités dans les cinq (5) régions du Sud du pays (Sikasso, Koulikoro, Ségou, Mopti, Kayes). Avec l'intervention des ONG membres du Groupe Pivot/Santé Population dans la DBC, et l'autorisation faite aux relais communautaires de fournir la première dose de pilule, en 6 mois, la prévalence est passée de 11 pour cent à 32 pour cent, voir 40 pour cent dans certaines zones, en particulier là où les ONG ont mis en œuvre la stratégie.

En 2003, la Direction Nationale de la Santé et ses partenaires, notamment le Groupe Pivot, ont relancé le programme de DBC. Le programme a recruté des hommes et des femmes agents de la communauté pour fournir entre autres des contraceptifs.

Autres projets documentées pour le Mali sont : 


\section{Programme USAID Santé Kènèya Ciwara Phases I et II (PUSKC I et PUSKC II)}

De 2004 à 2008, ce programme a visé à augmenter l'utilisation des services de santé communautaire de qualité à grand impact et à assurer l'adoption des pratiques familiales essentielles (PFE) au niveau des aires de santé fonctionnelles des 59 districts sanitaires du Mali, en s'assurant notamment de la capacité des Associations de Santé Communautaire (ASACO) de la zone d'intervention à soutenir l'éventail de services et la qualité des soins offerts dans le domaine de la santé maternelle et de la survie de l'enfant, y compris la PF.

Parallèlement, ce programme a travaillé au renforcement des capacités des prestataires et des organisations de la société civile impliquées dans la santé communautaire afin qu'ils améliorent la qualité de leurs services.

La deuxième phase (PUSCK II) a été mise en œuvre entre 2009 et 2011 et a été exécutée dans les districts sanitaires de Koutiala, Sikasso, Kadiolo et Bougouni de la région de Sikasso. Le renforcement des capacités des acteurs/trices en charge de la santé au niveau local a constitué la principale stratégie d'intervention du programme.

\section{Etude de faisabilité de l'insertion des implants par les matrones}

Suite à l'intégration dans les PNP, en 2012, de l'insertion des implants par les matrones, cette initiative a fait l'objet d'une étude de faisabilité avec l'appui du projet USAID/MCHIP. Cette étude a été menée en 2012 dans le district de Diéma de la région de Kayes. Elle avait pour but d'évaluer la faisabilité de l'insertion des implants par les matrones dans le cadre des services de soins de santé reproductive, maternelle et néonatale au niveau des centres de santé communautaire (CSCOM). 30 matrones ont reçu une formation de cinq (5) jours en prestation de service d'implants contraceptifs Jadelle (counseling, dépistage, insertion, référence pour le retrait et le respect de la prévention des infections).

Cette expérience a montré que les matrones peuvent insérer les implants de façon satisfaisante et a permis une augmentation de l'utilisation de la PF dans les centres où ces matrones exerçaient. Le nombre de nouvelles utilisatrices est passé de 114 en 2011 (avant la formation) à 688 après la formation en 2012, et à 773 en 2013. L'initiative a été jugée concluante et une décision a été prise de la répliquer dans le district de Kita (38 matrones formées en avril-mai 2013), et dans la région de Sikasso (105 matrones de cinq (5) districts formées en 2014).

\section{MAURITANIE}

Peu de documentation sur les expériences en matière de délégation des tâches en Mauritanie a pu être obtenue.

Un seul projet a pu être identifié. II s'agit du projet "Etendre l'accès à des services de santé sexuelle et reproductive de qualité ", mis en œuvre pendant cinq ans (de 2003 à 2007) par L'Association Mauritanienne pour la Promotion de la Famille (AMPF), avec une assistance technique de I'UNFPA, notamment pour l'évaluation finale. Le projet a été implanté dans six (6) localités urbaines et 50 zones rurales.

Le projet a établi quatre (4) cliniques mobiles pour couvrir 100 communautés rurales dans des zones jusquelà privées de tout service (dans les environs immédiats de Kif Fa, Rosso, Nouadhibou et Kaédi). Ces cliniques proposaient, entre autres services, diverses méthodes de contraception.

De plus, des réseaux d'hommes, de femmes et de jeunes, avec à leur tête un chef communautaire, ont été constitués dans chaque zone cible et formés. Ces groupes ont travaillé avec le personnel du projet pour promouvoir les droits sexuels de leur communauté et encourager l'utilisation des services. Les visites à 
domicile et le counseling individuel ont contribué à une nette évolution des attitudes et des connaissances. En effet, le projet a attiré 76917 nouvelles utilisatrices de méthodes contraceptives modernes et donné lieu à 68337 sessions de counseling individuel.

\section{NIGER}

Deux (2) expériences de délégation des tâches ont pu être identifiées.

\section{Projet Planning Familial/Prévention du SIDA}

Ce projet s'inscrit dans le cadre du Programme Régional Santé de la Reproduction et Prévention du VIH de I'espace CEDEAO sur la base d'un accord entre la DSME/MSP et I'ANIMAS-SUTURA. II est financé par l'Organisation Ouest Africaine de la Santé (OOAS).

L'approche de promotion de la santé développée par l'ANIMAS-SUTURA est construite sur des actions de CCC et de DBC. ANIMAS-SUTURA exécute depuis 2011 des sous-contrats/programmes annuels en vue de renforcer les activités de promotion de SSR dans les régions de Tillabéri et de Maradi et de booster l'utilisation des méthodes contraceptives modernes. Le programme a permis de former 654 agents DBC issus de 327 villages et d'effectuer des tournées de mobilisation sociale en faveur de la PF dans ces villages. Les sessions de formation ont visé l'introduction de la pilule contraceptive "Sutura " dans la DBC. Elles ont été dispensées par deux formatrices de la Direction Régionale de la Santé Publique (DRSP) de Maradi préalablement formées au module de formation des agents DBC.

A l'issue des formations, les agents DBC ont été dotés de mallette contenant des échantillons de contraceptifs aux fins de sensibilisation. Pour faciliter le marketing social de la pilule "Sutura " par les agents DBC, chaque village a été doté d'une cartouche (soit 54 plaquettes) en guise de fonds de roulement.

Pour investir les agents DBC dans leur nouveau rôle de promoteur de PF et en vue de susciter davantage de dialogue au niveau communautaire sur les enjeux de la pratique contraceptive, des tournées foraines ont été entreprises dans chaque village. Des séances de projections du film "Sutura Kariya " appuyées de discussion, et des consultations foraines de PF ont été réalisées. Ces activités ont été menées avec les radios communautaires et les chefs des Centres de Santé Intégrés (CSI) qui sont les superviseurs de proximité des agents DBC.

En fin 2013, tous les 327 villages DBC vendaient deux (2) produits contraceptifs de marketing social : la pilule "Sutura" et le condom "Foula ". Parmi les activités de l'année 2013, figure la vente de 14707 unités de condoms " Foula " et de 32593 cycles de pilule "Sutura ".

\section{Projet Recherche Action Plaidoyer (RAP)}

Le projet RAP, mené par Médecins du Monde (MDM) de 2007 à 2010, visait à améliorer les connaissances, attitudes et pratiques en matière de nutrition et de PF dans la région de Tahoua (districts de Keita, Abalak, Tchintabaraden) et à contribuer à diminuer la vulnérabilité des populations rurales par la mise en œuvre d'activités à base communautaire adaptées et reproductibles et d'actions d'IEC pour un changement de comportement sur les questions de nutrition et de PF.

Les activités suivantes ont été menées par des RC : sensibilisation aux méthodes modernes et naturelles de contraception, animation de causeries, counseling auprès de femmes, promotion de l'espacement des naissances, orientation vers un centre de santé pour recevoir une prescription. Dans les villages éloignés des structures sanitaires, les RC assuraient la distribution des contraceptifs après une première prescription au niveau du centre. Parmi les RC figuraient des femmes et des hommes, afin de mieux promouvoir l'implication de ces derniers sur les questions de SR et de PF. 
Par ailleurs, la disponibilité des intrants étant un des facteurs limitant de l'accès à la contraception, MDM disposait d'un stock-tampon de contraceptifs pour pallier les ruptures occasionnelles dans les centres de santé.

Les taux de prévalence contraceptive 2008-2009 ont augmenté dans les trois (3) districts d'intervention du projet. Ces résultats ont été attribués à la sensibilisation, à la DBC et aux activités foraines et mobiles. Ainsi, à l'occasion de la revue du Plan Annuel des Activités 2009 et de l'Evaluation 2010, le Directeur régional de la santé publique de Tahoua a fortement incité les équipes cadre de district (ECD) des huit (8) districts de la région de Tahoua à promouvoir la DBC de contraceptifs par les RC.

\section{SENEGAL}

Diverses initiatives de délégation des tâches ont été expérimentées au Sénégal.

\section{Counseling initial en planification familiale par des matrones au niveau des points de prestation de services}

Dans les années 1990, dans le but de décharger les sages-femmes et leur permettre de se concentrer sur des tâches plus cliniques, le Ministère de la Santé (MS) a lancé la formation des matrones en counseling en PF. Les résultats positifs de cette expérience ont encouragé le MS à faire passer cette initiative à l'échelle dans l'ensemble du système. De nos jours, l'éducation et le counseling initial des femmes en SR sont effectuées par les matrones formées sous la supervision de sages-femmes ou d'infirmières. Ces conseillères en PF sont disponibles dans la majorité des établissements de santé du pays.

\section{Projet DBC des contraceptifs de barrière}

En 1996, l'ONG Action et développement (ACDEV) a mis en œuvre un projet de DBC de méthodes contraceptives de barrières (préservatifs et spermicides). Cependant, les informations disponibles sur cette initiative ne sont pas suffisamment détaillées pour pouvoir la documenter.

Etude expérimentale sur l'offre de la distribution à base communautaire des services de santé de la reproduction au Sénégal. Une étude de cas dans le district sanitaire de Kébémer.

Cette étude a été mise en œuvre en 2004 par le Population Council. Les interventions comprenaient le développement et l'introduction de deux modèles de DBC dans le district de Kébémer:

- Un modèle qui consistait à utiliser les ASC, qui travaillaient dans 15 cases de santé communautaire. Pour le fonctionnement de ces cases, des fournitures supplémentaires et équipements ont été fournis suivant une évaluation des besoins. Deux (2) ASC ont été sélectionnés dans chaque case. Les 30 ASC ont reçu une formation de deux (2) semaines pour devenir agents de DBC.

- L'autre modèle a consisté en la création d'un nouveau type de travailleurs communautaires appelé Relais Communautaires (RC). Ils ont été utilisés là où une case de santé existait mais n'était pas fonctionnelle. Deux (2) RC (un homme et une femme) ont été sélectionnée. Les 30 RC ont reçu la même formation que les ASC.

Les activités consistaient en des visites à domicile, des entretiens sur des questions de SR, et la fourniture des services dans la communauté, la vente des produits contraceptifs (préservatifs et spermicides), et la référence vers le poste de santé. 
Les résultats d'évaluation indiquent que la performance des RC était supérieure à celle des ASC. En moyenne, les RC ont servis 1,35 fois plus de clients pour les produits contraceptifs et ont fait 1,39 fois plus de références que les ASC.

\section{Projet Services à Base Communautaire de Santé de la Reproduction (SBC/SR)}

Le projet SBC/SR a été mis en œuvre avec l'appui de I'UNFPA et du Population Council dans les régions de Tambacounda et de Kolda, plus exactement dans les trois districts cibles de Kédougou, Goudiry et Vélingara, afin de promouvoir les changements de comportements pour une meilleure santé de la reproduction/santé sexuelle dans l'optique d'une réduction de la mortalité maternelle et néonatale. 168 ASBC ont été formés pour acquérir des connaissances et des compétences en matière de services à base communautaire parmi lesquels la PF.

Le projet a duré 16 mois, de mai 2006 à août 2007. Pour l'évaluer, un procédé de recherche quasiexpérimental a été utilisé. Une étude de base sur les Connaissances Attitudes et Pratiques (CAP) a été réalisée avant le démarrage de l'intervention (en juillet 2005) pour permettre d'identifier le niveau des indicateurs clés et fournir les informations utiles pour le développement des stratégies par rapport à I'Information/Education/Communication (IEC) et à l'offre de services SR. Une deuxième enquête CAP a été exécutée à la fin de l'intervention (en novembre 2007). Ces deux enquêtes ont principalement concerné les cinq (5) composantes que recouvre la notion de services à base communautaire telle que retenue par le Ministère de la santé à savoir : la maternité à moindre risque, la PF, les IST y compris le Sida, la survie de l'enfant et les pratiques néfastes à la santé de la mère et de l'enfant.

Les rapports d'activités montrent que les ASBC ont réalisé dans le cadre des activités IEC, en moyenne par mois : deux (2) entretiens/counseling, deux (2) visites à domicile, deux (2) causeries éducatives et deux (2) référence. Les 168 ASBC des trois districts ont référé plus de 1700 personnes en 2006 (en huit (8) mois d'activités) et plus de 2600 personnes en 2007 (pour la même période d'activité) pour des motifs principalement liés aux CPN, aux accouchements, et à la PF.

Les résultats en matière de vente de produits montrent que les ASC constituent une source importante d'approvisionnement en produits contraceptifs et médicaments essentiels. Toutefois, les condoms sont plus demandés que les pilules et les spermicides.

Le pourcentage de femmes qui ont utilisé une méthode contraceptive moderne au cours des 12 derniers mois est passé de 9 pour cent au démarrage de l'intervention SBC à 12 pour cent après les 12 mois d'expérimentation dans les districts de Kédougou et de Vélingara. Dans le district de Goudiry, cette proportion est passée de 5 pour cent à 7 pour cent.

\section{Offre de méthodes à longue durée d'action au niveau des postes de santé}

Au cours de la période 2006-2011, l'ONG Intrahealth a expérimenté une initiative de transfert de tâches qui consistait à permettre aux Infirmiers Chefs de Poste (ICP) de fournir des méthodes de longue durée d'action (MLDA), à savoir les implants et DIU, au niveau du poste de santé. Ces méthodes n'étaient auparavant disponibles que dans les centres de référence, à savoir les centres de santé et les hôpitaux, et étaient administrés par des médecins ou sages-femmes. Sur la base de normes et protocoles pour la prestation de services de PF, les ICP n'avaient pas été autorisés à offrir ces méthodes, et celles-ci n'avaient pas été inclues dans leur programme de formation. L'intervention a couvert plus de 600 postes de santé. L'évaluation de cette initiative a indiqué que les ICP formés pouvaient offrir ces méthodes en toute sécurité. Les MLDA sont maintenant disponibles au niveau du poste de santé dans 12 des 14 régions du Sénégal (à l'exception de Matam et Tambacounda). 


\section{Programme de Santé de l'USAID/Santé Communautaire (PSSC)}

Ce programme mis en œuvre par Childfund répond à la nécessité exprimée par le Ministère de la Santé et de l'Action Sociale (MSAS) d'accorder la priorité à la répartition équitable de l'offre de services au niveau communautaire. Dès sa première phase (PSSC I), de 2006 à 2011, il visait, à travers l'intégration des services, une extension de l'accès à un paquet de base de soins primaires de santé, y compris la PF, dans les communautés rurales, par la redynamisation et l'équipement des cases de santé. Bien que celles-ci ne soient pas financées par le MSAS, elles sont considérées comme la base de la pyramide sanitaire au Sénégal. Le soutien du programme, pour chaque case, a consisté à recruter et former les acteurs communautaires.

Pour le PSSC II (2011-2016), ChildFund et ses partenaires (Africare, Catholic Relief Services, Plan International, Word vision, Enda Sahel et Enda Santé) se sont engagés à étendre dans tous les 14 régions du Sénégal les services de santé communautaire.

Les principales réalisations en matière de PF concernent le passage à l'échelle de l'offre initiale de pilule et l'extension de la couverture en contraceptifs injectables.

- Offre Initiale de Pilule dans les cases de santé (OIP)

En 2008, cette intervention pilote a couvert huit (8) régions (Fatick, Kaffrine, Kaolack, Kolda, Louga, Sédhiou, Thiès et Ziguinchor). Un total de 206 matrones et ASC ont été formées à la prestation sécuritaire de la première offre de pilule. Au cours de la mise en œuvre, aucune difficulté n'a été notée et les matrones et ASC étaient en mesure de répondre de manière adéquate aux effets secondaires signalés par les utilisatrices. La plupart des clientes ont exprimé leur satisfaction pour les services reçus.

L'évaluation a révélé que lorsque les matrones et ASC sont bien formées, elles peuvent effectuer l'administration sûre et correcte de pilules au niveau communautaire. Les données de l'évaluation à miparcours ont montré que le nombre de femmes utilisant la pilule a augmenté de 502 en 2008 à 5141 en 2009. Entre 2008 et 2009, le nombre d'utilisatrices ravitaillées en pilule a augmenté de 127 à 3 187, et le nombre d'abandons a diminué de 180 à 153 durant la même période. Le nombre de cases de santé qui offrent l'OIP a connu une progression constante depuis la fin de PSSC I. Ce nombre est passé à 624 cases en 2011 et à 1250 en septembre 2013. Les matrones ont adhéré aux procédures de consultation et ont été en mesure de remplir correctement les formulaires de collecte de données. Basé sur les résultats de cette expérience pilote, l'intervention a été considérée comme une meilleure pratique. Les enseignements positifs tirés ont mené le MSAS à porter à l'échelle l'initiative dans tout le pays. Les matrones et ASC ont montré qu'elles pouvaient offrir la pilule dans leur communauté de manière sûre et efficace.

- Offre initiale de Contraceptifs Injectables Intramusculaire (IM) dans les cases de santé

En 2012, PSSC II a appuyé la mise en œuvre d'une étude d'acceptabilité et de faisabilité de l'offre initiale de contraceptifs injectables en intramusculaire (DMPA-IM) par les matrones et ASC au niveau de 44 cases de santé des districts de Nioro dans la région de Kaolack, Dioffior dans la région de Fatick et Joal dans la région de Thiès, sous la coordination de la Direction de la santé de la reproduction et de la survie de l'enfant (DSRSE) et en partenariat avec FHI360/Progress et le CEFOREP. Au total 45 matrones et ASC ont été formées.

Les résultats de l'évaluation ont montré que les matrones et ASC ont développé les compétences nécessaires pour fournir correctement le DMPA-IM. La fourniture du DMPA-IM par des matrones et ASC a contribué à la diversification des méthodes offertes au niveau des cases de santé. Les matrones ont servi 1078 clients, dont 670 ont choisi les injectables. Les clients ainsi que d'autres personnels de santé ont apprécié cette expérience de délégation des tâches. 
- Offre initiale de Depo SubQ Uniject (Sayana® Press) dans les cases de santé

Le contraceptif injectable en Uniject a également fait l'objet en 2012 d'une étude d'acceptabilité auprès des clientes de PF, les prestataires des structures de santé et un échantillon de matrones de cases dans les districts de Thiès, Tivaouane et Mbour avec l'appui de PATH, FHI 360/Progress, PSSC II, et le CEFOREP. L'étude a été concluante et une décision de passage à l'échelle a été prise par la DSRSE.

Les résultats de l'évaluation ont montré que les matrones et ASC formés pouvaient administrer en toute sécurité le depo-subQ Uniject. Aucun événement indésirable n'a été signalé. Les clients ont montré un intérêt dans la méthode et ont confirmé le potentiel du Depo-subQ à accroître l'accès à la PF par la DBC. Les clients ont trouvé la méthode acceptable de même que la prestation de services par des matrones. Après cette expérience positive, le Ministère de la Santé a signé en août 2014 une note officielle pour étendre l'initiative à travers le pays comme indiqué par le Plan national sur les meilleures pratiques. Les régions de Dakar, Fatick et Kaolack mettront en œuvre des activités semblables en 2015.

\section{Offre communautaire de pilules et d'injectables par les relais communautaires des ONG ACDEV et ASBEF}

II s'agit d'une initiative mise en œuvre conjointement par l'Association Sénégalaise de Bien-Etre Familial (ASBEF), dans le district de Koumpentou depuis 2012, et l'ACDEV, dans le district de Guédiawaye depuis 2013. Elle consiste à expérimenter l'offre initiale de services de PF (pilule) par les RC dans leurs sites d'intervention.

Suite à l'identification de clientes potentielles lors d'activités de causeries, mobilisations sociales, VAD et niches ${ }^{15}$, les RC font une VAD pour un entretien plus approfondi ou le counseling initial afin de discuter avec la femme sur les avantages de la PF et lui présenter l'ensemble des méthodes pour un choix libre et éclairé. Si le choix de la cliente porte sur la pilule, une checklist lui est administrée et si elle présente les conditions requises pour la contraception, elle reçoit sa première plaquette de contraceptifs oraux ${ }^{16}$. Si le choix porte sur une autre méthode, la cliente est orientée vers une structure de santé en vue d'une consultation et la prescription par une sage-femme. Les clientes recrutées font l'objet d'un suivi régulier par les RC ou ASBC. Ce suivi permet de rappeler aux femmes le respect de la prise des contraceptifs, de les réapprovisionner en temps opportun afin de ne pas courir le risque de ruptures et partant, de démotivation ou de désintérêt.

Les résultats de l'expérience se sont révélés concluants et la perspective d'un passage à l'échelle est en cours d'examen avec un élargissement des méthodes offertes.

\section{TOGO}

Il est à noter que la DBC de méthodes contraceptives, y compris les injectables, par les ASC est inscrite au Plan de Travail Annuel (PTA) 2012 sous financement des fonds français de l'initiative MUSKOKA et du programme AWARE II/USAID. Deux expériences de délégation de tâches ont pu être documentées à savoir :

\section{Projet PF/PCIMNE-C}

En 2011, le Ministère de la Santé (MS) a pris l'initiative de tester le modèle de délégation des tâches à travers l'intégration du paquet PF au projet " Prise en Charge Intégrée des Maladies du Nouveau-né et de l'Enfant au niveau Communautaire (PCIMNE-C) ", dans les districts pilotes de Haho et Blitta, grâce à un partenariat avec le projet USAID AWARE II, I'ATBEF et I'ONG locale Appui au Développement et à la Santé communautaire (ADESCO). Ce projet a ciblé 158 villages. USAID AWARE II et l'OOAS ont fourni une assistance technique à

15 Cette activité communautaire se déroule sous forme de scène théâtrale sur un thème bien précis suivi de questions-réponses.

16 Pour l'instant l'offre initiale de Sayana press n'est pas effective pour des raisons administratives et logistiques. Toutefois, le processus est en cours pour inclure l'offre du Sayana press dans le paquet de services du relais. 
I'ATBEF et à l'ADESCO pour la formation de 432 ASC dans les aspects techniques de la prestation de services (bonne administration des contraceptifs).

Les principales activités de délégation des tâches développées ont été la mobilisation communautaire, I'injection de Depo Provera, la distribution de pilules, préservatifs masculins et féminins, et la référence de cas. L'évaluation a montré que parmi les 6554 injectables administrées par les ASC, un seul cas d'abcès provoqué par l'injection a été enregistré.

Dès que les ASC sont devenus opérationnels, l'utilisation des services de PF a augmenté de manière rapide. Par rapport aux centres de santé, les ASC ont atteint six (6) fois plus de femmes à Haho et huit (8) fois plus de femmes à Blitta (11 007 nouvelles utilisatrices de PF pour les ASC contre 2246 pour les centres de santé).

Une seconde phase du projet (2012-2013) a été mise en œuvre dans le district de Haho par l'ATBEF. Le nombre de nouvelles acceptrices au niveau communautaire a été de 12460 contre 1437 pour le district sanitaire.

\section{Programme de coopération entre I'UNFPA, I'UNICEF et l'OMS et le Ministère de la Santé et l'ONG ADESCO}

En 2012, un programme de coopération a été établit entre I'UNFPA, I'UNICEF et l'OMS d'une part et le Ministère de la Santé à travers la Direction de la Santé Familiale (DSF) et l'ONG ADESCO d'autre part pour permettre de répliquer l'expérience réussie de la DBC de méthodes contraceptives dans d'autres districts du Togo. Ce partenariat a fait en même temps le suivi des acquis du précédent projet exécuté par l'ONG dans le district de Blitta.

\section{Tableau synoptique des méthodes contraceptives offertes par les agents de santé communautaires dans les pays du Partenariat de Ouagadougou}

Le Tableau 4 donne une vue d'ensemble des méthodes contraceptives offertes par les agents de santé communautaires dans les pays du Partenariat de Ouagadougou.

Tableau 4 : Méthodes contraceptives distribuées par les agents de santé communautaires par pays

\begin{tabular}{|c|c|c|c|c|c|c|c|c|c|}
\hline $\begin{array}{l}\text { Méthode } \\
\text { Pays }\end{array}$ & Bénin & $\begin{array}{l}\text { Burkina } \\
\text { Faso }\end{array}$ & $\begin{array}{l}\text { Côte } \\
\text { d'ivoire }\end{array}$ & Guinée & Mali & Mauritanie & Niger & Sénégal & Togo \\
\hline Pilule & $X^{1}$ & $X^{2}$ & $X^{2}$ & $X$ & $X$ & $X$ & $X^{3}$ & $X^{4}$ & $x^{5}$ \\
\hline Préservatifs $^{7}$ & $X$ & $X$ & $X$ & $X$ & $X$ & $X$ & $X$ & $X$ & $X$ \\
\hline Injectables & - & - & - & $x^{5}$ & $X$ & - & $X^{4}$ & $X^{4}$ & $x^{5}$ \\
\hline DIU & - & - & - & - & - & - & - & - & - \\
\hline Implants & - & - & - & - & $X^{6}$ & - & - & - & - \\
\hline $\begin{array}{l}\text { Méthodes } \\
\text { d'abstinence } \\
\text { périodique }\end{array}$ & $X$ & $X$ & $x$ & $x$ & $x$ & $x$ & $x$ & $x$ & $x$ \\
\hline $\begin{array}{l}\text { Contraception } \\
\text { d'urgence }\end{array}$ & - & - & - & - & - & - & $x$ & - & - \\
\hline $\begin{array}{l}\text { Méthodes } \\
\text { permanentes }\end{array}$ & - & - & - & - & - & - & - & - & - \\
\hline
\end{tabular}

1: Uniquement le réapprovisionnement au niveau des relais

3: Au niveau des ASC, pas des relais

5: Nécessite l'adoption d'une politique formelle

7: Masculin et féminin

2: Uniquement le réapprovisionnement

4: Au niveau des matrones et ASC des cases de santé

6: Matrones uniquement

- : Non applicable 
Au regard des nouvelles directives de l'OMS sur la délégation des tâches en matière de PF, il apparait que quelques pays du Partenariat (Guinée, Mali, Niger, Sénégal, Togo) sont en phase avec les recommandations, même si, pour certains, les activités ne sont pas toujours menées sur la base de politiques formelles (ex : cas du Togo, de la Guinée). Par contre pour d'autres pays, des mesures s'imposent, par exemple pour l'offre initiale de la pilule au niveau communautaire (Bénin, Burkina Faso, Côte d'ivoire), ou l'extension de l'offre de MLDA au niveau clinique (dans les cas où des compétences autorisées par le cadre règlementaire n'offrent pas les services. Ex : Mauritanie où les infirmiers n'offrent pour le moment ni le DIU ni les implants) voire au niveau communautaire (Seul le Mali autorise l'insertion des implants par les matrones). 


\section{CONTRAINTES A LA MISE EN CEUVRE DES ACTIVITES DE DELEGATION DES TACHES :}

Certaines contraintes ont été documentées dans le cadre de la mise en œuvre des différent(e) $s$ projets/activités de délégation des tâches, à savoir :

- $\quad$ La faiblesse de la motivation des ASC qui est souvent à l'origine de leur mobilité.

- $\quad$ Le déficit de supervision régulière des acteurs à la base.

- La complexité des outils de collecte que les ASC ont du mal à remplir compte tenu de leur niveau d'instruction (faible ou nulle). Ceci a pour corollaire des insuffisances dans l'élaboration des rapports d'activités (problèmes de complétude et de promptitude).

- La faible capitalisation des acquis des approches communautaires, conduisant à une perte/ méconnaissance des bonnes pratiques en la matière.

- $\quad$ Le mauvais état des routes et pistes, et l'inadaptation des moyens de transport

- $\quad$ Le chevauchement entre les activités des différents partenaires ;

- Spécifiquement pour le Burkina Faso: l'insuffisance de fonctionnement du Comité Technique National de coordination et de suivi de la contractualisation (la non-tenue des sessions de 2012 et 2013) ; et l'insuffisance de l'intégration de l'approche dans le système formel de santé notamment aux niveaux intermédiaire et périphérique, ce qui a favorisé la faiblesse du dispositif d'appui et de coordination à ces niveaux. 


\section{Leçons apprises}

L'analyse des différentes expériences de délégation des tâches a permis de relever que:

Par rapport à la mise en œuvre des interventions :

$\checkmark$ La DBC est un mécanisme essentiel de décentralisation des services SR/PF au niveau communautaire.

$\checkmark \quad$ La DBC est la forme de délégation des tâches la plus couramment documentée dans les pays du Partenariat de Ouagadougou, certains l'ayant même implémentée depuis plus de 2 décennies

$\checkmark$ Les séances de consultation foraine gratuites de PF participent à rapprocher davantage les populations des services de PF.

$\checkmark \quad$ La bonne collaboration entre les ONG qui mettent en œuvre l'approche de contractualisation, les autorités sanitaires et les autres secteurs de développement améliore la mise en œuvre des activités et les résultats.

$\checkmark \quad$ Les services offerts au niveau communautaire sont de qualité acceptable et grandement appréciés par les populations.

\section{Par rapport au rôle des agents de santé au niveau communautaire :}

$\checkmark$ Bien que la dénomination et la description des tâches de ces agents change selon les pays, ils représentent un maillon essentiel pour améliorer l'accès aux MLDA, compte tenu de leur implication dans le counseling et les causeries éducatives.

$\checkmark \quad$ L'éducation par les pairs facilite l'accès aux services pour les adolescents.

\section{Par rapport à la formation et à la supervision :}

$\checkmark$ Quand ils sont bien formés, les agents de santé au niveau communautaire peuvent offrir adéquatement un ensemble de services de santé primaire de base et peuvent traiter les questions et les plaintes des membres de la communauté.

$\checkmark \quad$ Lorsque les agents de santé au niveau communautaire sont bien formés et bien supervisés, ils sont capables d'offrir, outre leurs activités éducatives, des services de prise en charge communautaire des maladies de l'enfant, et de PF incluant les injections de Depo Provera en intramusculaire, et les implants.

$\checkmark \quad$ L'existence d'agents de santé au niveau communautaire formés, équipés et supervisés est une expression concrète du droit à l'espacement des naissances vis-à-vis des femmes rurales situées audelà du rayon de couverture des formations sanitaires. La formation, l'équipement et le soutien de ces agents constituent une pratique à haut impact pour la PF. 


\section{Par rapport à l'impact :}

$\checkmark \quad$ Les activités d'éducation et de sensibilisation accrue sur la PF menées par les agents de santé au niveau communautaire, ainsi que leur présence dans la communauté stimulent la demande et l'utilisation des services.

$\checkmark$ Grâce à leurs activités éducatives, les agents de santé au niveau communautaire ont la possibilité de surmonter les obstacles culturels qui limitent l'utilisation des services de santé par les individus et les familles.

$\checkmark \quad$ La fourniture de contraceptifs injectables par les agents de santé au niveau communautaire peuvent conduire à l'augmentation de l'utilisation des contraceptifs dans les zones à faible taux de prévalence contraceptive, celles ayant des niveaux élevés de BNS, et celles ayant un manque d'accès à une large gamme de méthodes et de services cliniques.

\section{Pour la pérennisation :}

$\checkmark$ Les motivations extrinsèque des agents de santé au niveau communautaire (certification de la compétence avant le démarrage des activités, remise officielle de certificat de reconnaissance de la performance en cours d'intervention, installation officielle par l'autorité administrative ou coutumière de la zone d'intervention, fourniture d'équipements, exemption des travaux communautaires, etc.) et intrinsèque (perdiem journalier lors des formations/recyclages, motivation financière mensuelle) peuvent, dans certains cas, aider à maintenir les agents de santé au niveau communautaire dans leur rôle et améliorer la qualité de leurs services.

$\checkmark \quad$ Lorsque le choix des agents de santé au niveau communautaire se fait de façon démocratique par leur communauté, cela facilite l'acceptation des SBC par la communauté, améliore la recherche de soins par les populations, motive les agents de santé au niveau communautaire et renforce leur maintien dans leur rôle. Cela renforce aussi la confiance de la communauté à l'ASC

$\checkmark \quad$ II y a des avantages géographiques et financiers à la délégation des tâches, tels que la réduction des déplacements, du temps et des coûts pour l'obtention de services de santé.

$\checkmark \quad$ L'implication des leaders communautaires, religieux et des groupes organisés est déterminante pour faciliter l'appropriation des initiatives par les communautés. 


\section{Recommandations}

Les recommandations suivantes ont été formulées pour que la délégation des tâches soit plus efficace.

\section{Sur le plan politique}

- Les gouvernements doivent renforcer l'élaboration de politiques nationales en faveur de l'introduction, de la poursuite et de l'extension de l'offre de méthodes contraceptives au niveau communautaire.

- Une politique novatrice et compréhensive de santé communautaire qui précise et clarifie le rôle des différents acteurs doit être élaborée dans les pays où elle n'existe pas encore.

- Un plaidoyer doit être fait pour la révision des politiques, normes et procédures afin de les adapter aux directives de l'OMS sur la délégation des tâches de PF.

- $\quad$ Les agents de santé moins qualifiés dans les formations sanitaires rurale doivent être formés et autonomisés pour offrir les MLDA.

- Les changements dans les politiques doivent être intégrés dans les documents de stratégies opérationnelles.

- Au plan législatif et réglementaire, le dispositif en rapport avec les nouvelles responsabilités assignées aux AC doit être mis à jour/adapté

- Un plan de passage à l'échelle pour les expériences réussies incluant un cadre de concertation/ coordination doit être proposé.

\section{Sur le plan opérationnel}

- Une bonne formation du personnel de santé communautaire doit rester un impératif dans tous les efforts de prestation de services. C'est pourquoi, le niveau de formation de base au recrutement de ce personnel doit être bien étudié de même que le contenu de la formation.

- Une supervision rigoureuse et formative doit être de mise pour veiller à la qualité des services offerts par les agents de santé au niveau communautaire, notamment en ce qui concerne l'offre des injectables.

- L'utilisation de seringues autobloquantes doit être promue pour accroitre la sécurité des injections par les agents de santé au niveau communautaire. Ces derniers doivent être correctement formés à leur utilisation et à leur élimination en toute sécurité.

- Les politiques de DBC doivent être mises à l'échelle. Ceci devra inclure la mise en place d'un système d'approvisionnement qui pourrait prévenir les ruptures de stock.

- L'offre de MLDA doit être promue à travers les infirmières auxiliaires/ brevetées et les matrones.

- Les adolescents et jeunes doivent être ciblés davantage par les activités d'IEC/CCC et l'offre de services

- Les agents de santé au niveau communautaire doivent être recyclés périodiquement pour améliorer leurs performances par rapport des nouvelles tâches qui leur sont confiées

- Un système d'information qui permet d'assurer une remontée efficace et systématique des données doit être mis en place. 


\section{Pour la pérennité des interventions}

- Les agents de santé au niveau communautaire doivent être motivés pour garantir leur maintien dans le cadre des projets/interventions de délégation des tâches.

- La formation et la supervision des agents de santé au niveau communautaire doivent être renforcées pour l'extension de l'offre de services de qualité dans les zones rurales

- Les gouvernements doivent élargir et diversifier les sources de financement, par exemple en augmentant le financement public, en attirant de nouveaux partenaires financiers et en impliquant plus le secteur privé et les organisations de la société civile.

- Les gouvernements, les ONG partenaires, et la communauté internationale doivent assurer un approvisionnement continu en médicaments et en produits de PF, et le soutien technique et financier approprié au bon déroulement des interventions.

- Les expériences et interventions exécutées dans le cadre de la délégation des tâches doivent être mieux documentées pour le partage de bonnes pratiques au niveau national, régional et international.

\section{Limites de l'étude}

La principale difficulté rencontrée au cours de cette étude a été l'accès à la documentation. La littérature portant sur certaines expériences de délégation des tâches dans les pays est quasi inexistante sur Internet. Ce sont surtout les sites des organismes de financement qui ont permis de collecter des informations sur les projets, mais celles-ci n'étaient pas suffisamment détaillées pour l'objectif de l'étude.

Les Points Focaux du Partenariat et d'autres acteurs-clé ont été contactés dans chaque pays pour aider à l'obtention d'informations. Cela a beaucoup aidé lorsque leur réactivité était bonne. Cependant, cela n'a pas toujours été le cas, malgré de nombreuses relances. 


\section{Conclusion}

Les programmes communautaires sont particulièrement importants pour réduire les inégalités d'accès aux services de PF. Un nombre croissant de données factuelles démontrent l'efficacité, la sécurité et l'acceptabilité de la délégation des tâches dans le domaine des services de SSR. La délégation des tâches se pratique d'ailleurs déjà à des degrés divers dans les pays du Partenariat de Ouagadougou, en réponse pragmatique aux pénuries d'effectifs de santé.

Les expériences en milieu communautaire documentées dans les pays du Partenariat de Ouagadougou démontrent que des effets bénéfiques peuvent être obtenus en lien avec l'amélioration de la santé maternelle et infantile. Cette étude donne également un éclairage sur les modèles novateurs testés au fil du temps pour augmenter la demande en PF et l'accès aux services.

La faisabilité et l'acceptabilité de la DBC des contraceptifs prouvent qu'un changement programmatique et politique pourrait améliorer l'accès des femmes aux services de PF. La DBC peut constituer une intervention pertinente et un mécanisme essentiel de décentralisation des services SR/PF au niveau communautaire pour les pays à faibles indicateurs d'accès à la PF.

Les expériences documentées ont permis de montrer que, dans le contexte Ouest-africain, il est possible pour des bénévoles communautaires bien formés et soutenus d'assurer une administration efficace et à moindre risque des contraceptifs hormonaux. Les résultats permettent également de confirmer dans le contexte Ouestafricain les constatations de I'OMS sur la base d'une consultation technique de juin 2009 selon lesquelles " avec une bonne formation axée sur les compétences, les ASC peuvent efficacement administrer les méthodes injectables en toute sécurité, et fournir des conseils appropriés sur les effets secondaires, en faisant preuve de compétences équivalant à celles des prestataires au niveau des formations sanitaires ". (OMS, DBC brief des conclusions de la consultation technique, 2009. P3).

La pertinence de l'agent de santé au niveau communautaire n'est plus un questionnement pour la plupart des bénéficiaires et des acteurs, bien que la question de leur rémunération demeure au cœur de leur pérennisation. Par ailleurs, la reconsidération de leur statut, leur formation et leur supervision seront essentiels pour l'extension de l'offre des services de qualité dans les zones rurales.

La poursuite et la pérennisation de ces activités dépendent toutefois de l'engagement des gouvernements, des ONG partenaires, et de la communauté internationale à assurer un approvisionnement continu en médicaments et en produits de PF et le soutien technique et financier approprié. 


\section{Références bibliographiques}

Advancing Partners \& Communities. 2013. Country Profile: Sénégal Community Health Programs. Arlington, VA: Advancing Partners \& Communities.

Advancing Partners \& Communities. 2014. Country Profile: Benin Community Health Programs. Arlington, VA: Advancing Partners \& Communities.

Advancing Partners \& Communities. 2014. Country Profile: Mali Community Health Programs. Arlington, VA: Advancing Partners \& Communities.

ARRETE N031/MSP/MDS/P/PF du 30 juillet 1992 Autorisant les agents de Santé villageoise à offrir certaines prestations en $\mathrm{PF}$

ASAPSU, Rapport de formation des animatrices communautaires en distribution à base communautaire en planification familiale, Abidjan, 2011.

Association de Soutien à I'Autopromotion Sanitaire et Urbaine (ASAPSU), Rapport d'activités de juillet à novembre 2011.

Association Guinéenne pour le Bien-être Familiale, Résultats de l'évaluation interne des activités de l'initiative onchocercose - santé de la reproduction, Guinée

ATBEF, Mise en œuvre du Paquet Intégré de planification familiale, de lutte contre le VIH/Sida, de promotion de la santé maternelle et de soins aux enfants au niveau communautaire dans le District Sanitaire de Haho au Togo, Rapport technique du premier trimestre (de juillet à Septembre 2011), Lomé, octobre 2001.

Bako Bagassa, Consolidation des acquis des financements 2011, 2012, et 2013 et Création de nouveaux sites à Filingué, Balleyara, Say, Torodi, Kornaka, et dans 30 villages additionnels de Tessaoua, Aguié, Guidan Roumdji, Dakoro, Mayahi et Gagama , ANIMAS-SUTURA, mars 2014.

Banda Ndiaye, Judi Aubel, Abdou Karim Guèye. Rapport évaluation " projet ATSC " Plan Guinée

Camille KUYU, 2008. La santé de la reproduction en Mauritanie: Analyse de l'environnement juridique et socioculturel, UNFPA.

Childfund \& Co, 2014. Santé Communautaire, Phase II, Plan d'action An 3, Oct. 2013- Sept 2014.

Childfund\&Co, 2013. Rapport annuel d'activités Oct. 2012- Septembre 2013,

ChildFund, 2011 : Expérience Acquise de Programme de Santé Communautaire ChildFund/USAID-Sénégal Évaluation Finale. Programme de Santé Communautaire du Sénégal/ChildFund 2006-2011. USAID et Initiatives Inc.

Conférence "population, développement et planification familiale en Afrique de l'ouest francophone : I'urgence d'agir". Ouagadougou, 8-10 février 2011.

Consortium: ETC, Ecorys, KIT, CDR. Pratique contraceptive et la contribution des activités de planification familiale au mali. Une étude dans le cadre de la coopération malienne - néerlandaise. Août 2012

Dieudonné Ouedraogo, Les dynamiques démographiques. Série population, Atlas de l'intégration régionale en Afrique de l'Ouest, novembre 2011. 
Diouratié SANOGO, Mady CISSE, Médecin-Colonel Adama NDOYE, Laty G. NDOYE, Ousmane Faye, Balla Mbacké MBOUP, 2004. Etude Expérimentale sur l'offre de la Distribution à Base Communautaire des Services de Santé de la Reproduction au Sénégal. Une étude de Cas dans le District Sanitaire de Kébémer. Population Council.

Direction De La Promotion De La Santé, Rapport d'avancement de la mise en œuvre de l'approche de contractualisation des services de santé à base communautaire avec les ONG/RENCAP et les OBC-E 2012 à 2013, avril 2014.

Direction de la santé de la mère et de l'enfant, 2012. Planification familiale au Niger : plan d'action 2012 2020 ;

Direction Nationale de la Santé, Division de la Santé de la Reproduction, Politiques et Normes des services de santé de la reproduction, Mali, juin 2013.

Division de la Santé de la Reproduction (DSR) et Ministère de la Santé et de l'Action Sociale. Plan d'Action National de Planification Familiale 2012-2015. République du Sénégal.

Division de la Santé de la Reproduction (DSR) et Ministère de la Santé et de l'Action Sociale. 2011. Plan Stratégique de la Santé de la Reproduction du Sénégal 2012-2015.

Division de la Santé Familiale, Politique et Normes en santé de la reproduction, planification familiale et infections sexuellement transmissibles du Togo, 2ème édition, Lomé, décembre 2009.

Dr Awa Seck, Dr Dieudonné Valéa, Analyse de la santé communautaire au Burkina Faso, février 2011.

DSFC, Politique et Normes des Services de Santé de la Reproduction Février 2000

DSFC, Procédures des Services de Santé de la Reproduction Février 2000

Futures Group, repositioning family planning in Mali: Status of family planning programs in Mali, August 2012.

IntraHealth International. 2012. Evaluation Rapide de la Planification Familiale au Bénin - Rapport d'étude. IntraHealth International.

James Foreit, Sarah Raifman, Increasing access to Family Planning (FP) and Reproductive Health (RH) services through task-sharing between Community Health Workers (CHWs) and community mid-level professionals in large-scale public-sector programs. A Literature Review to Help Guide Case Studies. Population Council, February 2011.

Julia Bluestone, JHPIEGO, Vers une redistribution des tâches pour une palette de compétences stratégiques, résumé technique 5, CapacityProject, juin 2006.

Maiga, M, Deme Diallo, B., McDavid, E. and Attama Dissirama, S. 2012. Repositioning Family Planning in Mali : A Baseline. Washington, DC: Futures Group and the William and Flora Hewlett Foundation.

Metangmo, Dr. Pierre-Marie. 2012. Partnership for the Community Management of Child Health (PRISE-C) Midterm Evaluation Report. Bethesda: Center for Human Services.

Ministère de la Santé et de l'Action Sociale, 2014. Plan National Stratégique Santé communautaire 20142018. République du Sénégal.

Ministère de la santé et de l'hygiène publique, Politique Nationale de la Santé communautaire, Guinée Décembre 2012

Ministère de la Santé et de I'Hygiène Publique. Plan d'Action National de Repositionnement de la Planification Familiale en Guinée 2014-2018

Ministère de la Santé et de la prévention. 2009. Plan National de Développement Sanitaire (PNDS) 20092018. République du Sénégal. 
Ministère de la Santé et l'Hygiène Publique Plan d'Action National de Planification Familiale du mali 20142018

Ministère de la Santé Publique, Plan d'Action Planification Familiale 2012-2020, juin 2012.

Ministère de la Santé Publique, Plan de Développement des Ressources Humaines 2011-2020 en Santé.

Ministère de la Santé, Direction des Soins de Santé de Base et de la Nutrition, Programme National de Santé de la Reproduction, Plan d'Action en faveur de l'espacement des naissances 2014-2018, Mauritanie.

Ministère de la santé, Direction de la planification, de la coopération et de l'information sanitaire Stratégie nationale de délégation des tâches en santé, 2013.

Ministère de la santé, février 2013. Plan d'action pour le repositionnement de la planification familiale au Togo 2013 - 2017.

Ministère de la santé, Plan National De Relance De La Planification Familiale 2013 - 2015, Burkina Faso, janvier 2013.

Ministère de la santé, Plan National de repositionnement de la PF (2014-2018), Bénin.

Ministère de la santé, Rapport d'avancement de la mise en œuvre de l'approche de contractualisation des services de santé à base communautaire avec les ONG/RENCAP et les OBC-E, 2012 à 2013.

Ministry of Health, Republic of Benin. 2010. Directives Nationales pour la Promotion de la Santé au Niveau Communautaire. Cotonou: Ministry of Health, Republic of Benin. Available at http://www.beninsante.bj/documents/DNSP/directives_nationales_communautaire.pdf $\quad$ (accessed December 2013).

Ministry of Health, Republic of Benin. 2010. Paquets d'Interventions à Haut Impact par Niveau de Soins pour l'Atteinte des OMD au Benin. Cotonou: Ministry of Health, Republic of Benin.

Ministry of Health, Republic of Benin. 2011. Cahier du Relais Communautaire. Cotonou: Ministry of Health, Republic of Benin.

Ministry of Health, Republic of Benin. 2011. Guide de l'Animateur Pour la Formation des Relais Communautaires sur le Paquet Complet d'Interventions au Niveau Communautaire. Cotonou: Ministry of Health, Republic of Benin.

Mohamed Lemine ould Mohamed El Hadj, Stratégie Nationale de Délégation des Tâches en santé(SNDT), Direction de la planification de la coopération et de l'information sanitaire, Ministère de la santé(Mauritanie), février 2013.

OMS, DBC brief des conclusions de la consultation technique, 2009.

Organisation des Nations Unies, Objectifs du Millénaire pour le développement, Rapport de 2013, 60p.

Organisation mondiale de la Santé 2007 Task Shifting, Global Recommendations and Guidelines

Organisation Mondiale de la Santé, 2010. Accroître l'accès aux personnels de santé dans les zones rurales ou reculées grâce à une meilleure fidélisation : recommandations pour une politique mondiale.

Organisation Mondiale de la Santé, 2012. "Optimisation des rôles du personnel de santé par la délégation des tâches pour améliorer l'accès aux interventions de santé maternelle et néonatale”. Décembre 2012.

Organisation Mondiale de la Santé, U.S. Agency for International Development, Family Health International (FHI). Les agents de santé communautaires peuvent administrer des contraceptifs injectables en toute sécurité et efficacité : conclusions d'une consultation technique. Research Triangle Park (NC): FHI; 2009. 
Organisation Mondiale de la Santé. Rapport sur la situation dans le monde 2006 : travailler ensemble pour la santé.

Partenariat de Ouagadougou. La planification familiale : l'Afrique de l'ouest francophone en mouvement. Un appel à l'action, 2011)

Phillips J. F., Greene W. L., Jackson E. F., Lessons from community-based distribution of family planning in Africa, New York : Population Council, 1999, Document de travail $\mathrm{n}^{\circ}$ 121, 105 pages. Disponible à l'adresse suivante (en anglais uniquement) : http://www.popcouncil.org/pdfs/wp/121.pdf

Politique et normes en santé de la reproduction, planification familiale et infections sexuellement transmissibles du Togo, 2009.

Population Reference Bureau, Family planning worldwide, 2008 data sheet.

Programme National de Santé de la Reproduction 2005-2009.

Projet Recherche Action Plaidoyer Niger, Médecins Du Monde. Rapport de mission d'évaluation interne, Février 2010.

Rapport technique, ANIMAS, 2éme trimestre 2013.

Rapport technique, ANIMAS, période du 1er janvier au 31 mars 2014

Sara Pacqué-Margolis and Amanda Puckett, Community Health Workers: Meeting the Unmet Need for Family Planning in West and Central Africa, May 2011.

Sarah Castle, Preventing Student Pregnancy in Guinea’s Forest Region, Plan Guinea, August 2009.

STEP UP: analyse des besoins non satisfaits de PF au Sénégal. Mars 2013

USAID AWARE II, Distribution à base communautaire des contraceptifs hormonaux en Afrique de l'ouest, Togo, juin 2012.

USAID, guide de supervision des agents de santé communautaire en Planification familiale (PF) et en Prise en Charge Intégrée des Maladies de l'enfant et du nouveau-né au niveau Communautaire (PCIMNE C).

WHO/Africa, Atlas of african health statistics 2014: health situation analysis of the african region.

Winnie Mwebesa, Save The Children's Experience with Referrals, Flexible Fund Partners' Meeting April 30 2013.

\section{Présentations}

Adji Kossi, Mise en œuvre du paquet intégré de PF/PCIMNE à base communautaire au Togo, ATBEF, avril 2013.

Anne Pfitzer, MHS - MCHIP Washington Mme Haidara, Aissata Tandina, Sage-femme d'Etat - MCHIP Mali. Etude sur la Dévolution des Tâches d'Insertion du Jadelle par les Matrones, Septembre 2013

Bilan de la mise en œuvre des activités 2013, BURCASO.

Expériences des ONG RENCAP en matière de Burkina Faso, Atelier Régional de Fin de Projet AWARE II, $12-13$ Juin 2012.

La distribution à base communautaire des contraceptifs au burkina faso, Présentation introductive de la DSME, Rencontre bilan 2011 de mise en œuvre Ouagadougou, avril 2012.

Maiga, M, Deme Diallo, B., McDavid, E. and Attama Dissirama, S. 2012. Repositioning Family Planning in Mali: A Baseline. Washington, DC: Futures Group and the William and Flora Hewlett Foundation. 
Mamady Kourouma, Expérience de la Guinée en matière de délégation des tâches, 3e Conférence Internationale sur la planification Familiale, Addis Abeba, 11 novembre 2013.

Plan d'Action National de Planification Familiale du Mali 2014-2018

Plan Stratégique National de la Santé de la Reproduction, Mali, 2014-2018.

\section{EDS}

Agence Nationale de la Statistique et de la Démographie (ANSD) et ICF International. 2012. Enquête Démographique et de Santé à Indicateurs Multiples au Sénégal (EDS-MICS) 2010-2011. Calverton, Maryland, USA: ANSD et ICF International.

Agence Nationale de la Statistique, Enquête démographique et de santé Continue au Sénégal (EDS-Continue) 2012-2013. Calverton, Maryland, USA: ANSD et ICF International.

Cellule de Planification et de Statistique (CPS/SSDSPF), Institut National de la Statistique (INSTAT/MPATP), INFO-STAT et ICF International, 2014. Enquête Démographique et de Santé au Mali 2012-2013. Rockville, Maryland, USA : CPS, INSTAT, INFO-STAT et ICF International.

Direction Générale de la Statistique et de la Comptabilité Nationale, et ICF International. 2014. Troisième Enquête Démographique et De Santé du Togo (EDST-III) Rapport Préliminaire. Rockville, Maryland, USA : DGSCN et ICF International.

Direction Nationale de la Statistique (DNS) Enquête Démographique et de Santé, Guinée 2005. (2005). (Guinée) et ORC Macro. Calverton, MD: DNS and Macro International Inc.

Enquête Démographique et de Santé et à Indicateurs Multiples du Burkina Faso 2010. Calverton, Maryland, USA : INSD et ICF International.

Enquête par Grappes à Indicateurs Multiples, 2011. Suivi de la situation des femmes et des enfants, Mauritanie.

Institut National de la Statistique (INS) et ICF International, 2013. Enquête Démographique et de Santé et à Indicateurs Multiples du Niger 2012. Calverton, Maryland, USA : INS et ICF International.

Institut National de la Statistique (INS) et ICF International. 2012. Enquête Démographique et de Santé et à Indicateurs Multiples de Côte d'Ivoire 2011-2012. Calverton, Maryland, USA : INS et ICF International.

Institut National de la Statistique et de l'Analyse Économique (INSAE) et ICF International, 2013. Enquête Démographique et de Santé du Bénin 2011-2012. Calverton, Maryland, USA : INSAE et ICF International.

Office National de la Statistique (ONS) [Mauritanie] et ORC Macro. 2001. Enquête Démographique et de Santé Mauritanie 2000-2001. Calverton, Maryland, USA : ONS et ORC Macro.

Office National de la Statistique (ONS), 2011. Enquête Démographique et de Santé MICS 4, Mauritanie 2011. 


\section{Sites web et articles web}

Accès universel à la santé sexuelle et reproductive au Burkina : L'espoir se trouve dans les services à base communautaire, disponible sur http://www.lefaso.net/spip.php?article49061, consulté le .8 septembre 2014.

Guttmacher Institute, "Costs and Benefits of Investing in Contraceptive Services in the Developing World," June 2012. https://www.guttmacher.org/pubs/FB-Costs-Benefits-Contraceptives.html

http://partenariatouaga.org/

http://sites.path.org/rh/recent-reproductive-health-projects/sayanapress/ http://www.afd.fr/jahia/webdav/site/afd/shared/PORTAILS/SECTEURS/SANTE/pdf/Fiches\%20projets\%2 02013/CCI\%201202.pdf

http://www.afd.fr/jahia/webdav/site/afd/shared/PORTAILS/SECTEURS/SANTE/pdf/Fiches\%20projets\%2020 13/CCl\%201202.pdf.

http://www.fhi.org/en/research/projects/progress/gtl/concba2i.htm.

Intensification de la politique de planification familiale : Permettre un saut quantitatif de la couverture de la planification familiale en améliorant l'accessibilité de la population à des services de qualité et à réduire la mortalité maternelle:

Pratiques à Haut Impact dans la Planification Familiale (HIP). Agents de santé communautaires : apporter les services de planification familiale là où vit et travaille la population, Washington : USAID, oct. 2012. Disponible à l'adresse suivante : www.fphighimpactpractices.org/resources. 


\section{POPULATION COUNCIL}

Ideas. Evidence. Impact.

popcouncil.org

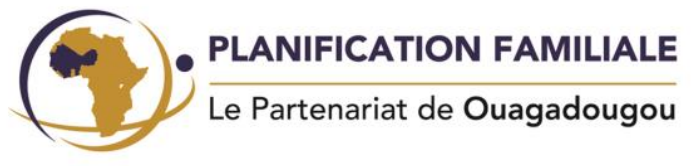

partenariatouaga.org 